\title{
Structure of Defects and Microstructure Evolution in Oxide Ceramics - Role of Electronic Excitation and Selective Displacement Damage
}

\author{
Kazuhiro YASUDA ${ }^{1}$ \\ ${ }^{1}$ Department of Applied Quantum Physics and Nuclear Engineering, Kyushu University (Fukuoka, JAPAN)
}

Fluorite- and spinel-type oxide ceramics, such as yttria-stabilized zirconia, ceria, magnesium aliminate spinel, are known to be exceptionally resistant to irradiation damage with energetic particles. Those oxide compounds, thereofore, have potential applications to advanced nuclear fuels and innert matrices of transmutaion targets. Fundamental understanding of radiation damage is apparently essential to assess the microstructure stability of fuel/target materials under the hostile environment. In this presentation, structure and stability of radiation-induced defects in the oxide ceramics is reported. Transmission electron microscopy (TEM) and scanning transmission electron microscopy (STEM) is utilized to examine the following topics focucing on role of electronic excitation and selective displacement damage: (1) effects of selective displacement damage of oxygen sublattice in fluorite-type oxides, such as stabilided cubic $\mathrm{ZrO}_{2}, \mathrm{CeO}_{2}$ [1-3], (2) atomic structure of ion tracks in $\mathrm{CeO}_{2}$ and $\mathrm{MgAl}_{2} \mathrm{O}_{4}$ induced by swift heavy ions, and microstructure evolution at high fluences under overlapping irradiation of high density electronic excitation damage [4-6], and (3) production and stability of defects in $\mathrm{MgAl}_{2} \mathrm{O}_{4}$ and alumina $\left(\alpha-\mathrm{Al}_{2} \mathrm{O}_{3}\right)$ under synergistic irradiation with dispacement damage and electronic excitation [7].

It is noted in fluorite-type oxides that there exists a significant difference in mass between cations and anions, and that the displacement energy of cation-sublattice is larger than the anion one. This leads to a higher displacement damage rate or selective displacement damage in oxygen sublattice in fluorite-type oxides. Figure 1 shows examples of TEM images in $\mathrm{CeO}_{2}$ showing dislocation loops formed by electron irradiation with energies ranging from 200 to $1250 \mathrm{keV}$ [1]. From contrast analysis and atomic resolution observations with TEM and STEM, those defects are found lying on $\{111\}$ planes and have been discussed to be composed of solely oxygen ions formed under the selective displacement damage [1]. Similar defects were formed in yttria stabilized $\mathrm{ZrO}_{2}$, in which entirely different growth process were observed compared to the normal (perfect) dislocation loops [2]. Molecular dynamic simulations in $\mathrm{CeO}_{2}$, which showed the recovery process of multiple oxygen Frenkel pairs, revealed a tendency of oxygen interstitials for clustering on $\{111\}$ planes, supporting the interpretation from the TEM and STEM observations [3].

Atomic resolution TEM and STEM observations in $\mathrm{MgAl}_{2} \mathrm{O}_{4}$ and $\mathrm{CeO}_{2}$ irradiated with $200 \mathrm{MeV} \mathrm{Xe}$ ions have shown that the crystal structures are retained at the core region of ion tracks, showing their excellent resistance to high density electronic excitation [4,5]. Figure 2 is an example of atomic resolution image of ion tracks in $\mathrm{CeO}_{2}$ with annular bright-field (ABF) STEM technique, showing that the crystal structure of $\mathrm{Ce}$-cation column is retained at the core region of ion tracks, whereas O-anion column is preferentially distorted at the core region of ion tracks [5]. The atomic density inside the core damage region of ion tracks in both crystals was found to be decreased, which suggests the existence of high density of vacancies and/or small vacancy clusters inside the ion tracks [5]. Overlap of ion tracks at high fluence was found to develop dislocation structure in both $\mathrm{MgAl}_{2} \mathrm{O}_{4}$ and $\mathrm{CeO}_{2}$. The development of dislocation structure also results in the formation of small sub-grains in $\mathrm{CeO}_{2}$. Those microstructure evolution was discussed with the generation of interstitial atoms at the peripheral region of the core damage region of ion tracks $[4,6]$.

It has been shown in $\mathrm{MgAl}_{2} \mathrm{O}_{4}$ and $\alpha-\mathrm{Al}_{2} \mathrm{O}_{3}$ that synergistic irradiation with displacement damage and electronic excitation retards the nucleation of dislocation loops and enhance their growth [7]. Electronic excitation with $200 \mathrm{keV}$ electrons makes small dislocation loops of interstitial-type unstable in $\mathrm{MgAl}_{2} \mathrm{O}_{4}$ and $\alpha-\mathrm{Al}_{2} \mathrm{O}_{4}$. Dislocation loops were found to be disappeared during electron irradiation. Analysis of the temperature dependence of the elimination process leads to a conclusion that the 
dislocation loops are disappeared by dissociating into isolated interstitials. The elimination rate of loops was higher in $\mathrm{MgAl}_{2} \mathrm{O}_{4}$ than $\alpha-\mathrm{Al}_{2} \mathrm{O}_{3}$, which indicates the high sensitivity of $\mathrm{MgAl}_{2} \mathrm{O}_{4}$ to electronic excitation, and this is considered to play a role for the radiation resistance of $\mathrm{MgAl}_{2} \mathrm{O}_{4}$.
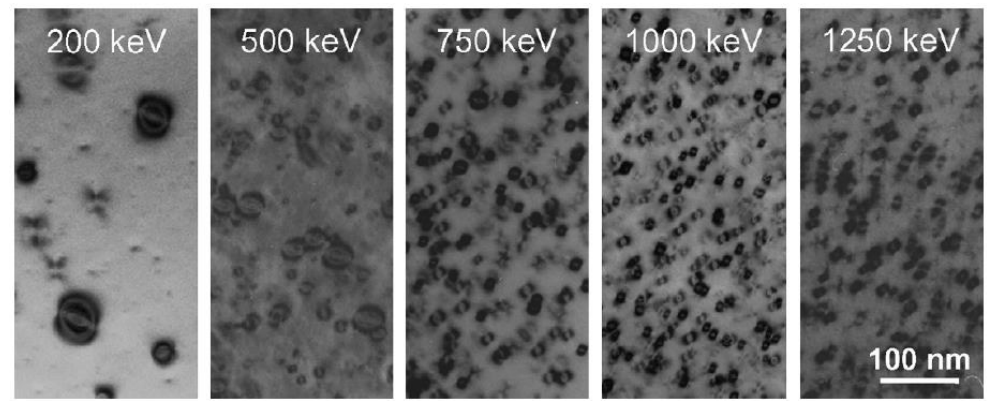

Fig. 1: Dislocation loops formed by electron irradiation ranging from 200 to $1250 \mathrm{keV}$ at room temperature. Dislocation loops are considered to be interstitial-type loops consist of oxygen ions on $\{111\}$ planes.

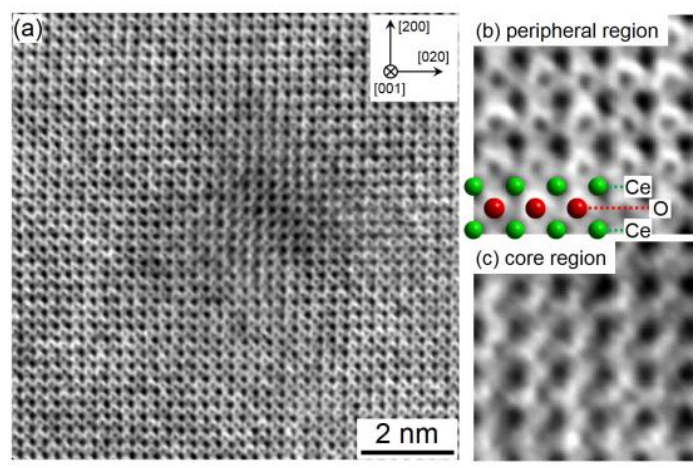

Fig. 2: Annular bright-field (ABF) images of ion tracks in $\mathrm{CeO}_{2}$ irradiated with $200 \mathrm{MeV} \mathrm{Xe}$ ions to a fluence of $3 \times 10^{12} \mathrm{~cm}^{-2}$. An ion track is nearly at end-on condition parallel to [001] direction.

\section{References}

[1] K. Yasunaga, K. Yasuda, S. Matsumura, T. Sonoda, 'Electron-energy dependent formation of dislocation loops in $\mathrm{CeO}_{2}$ ', Nucl. Instr. and Meth. B 266 (2008) 2877.

[2] K. Yasuda, C. Kinoshita, S. Matsumura, A.I. Ryazanov, 'Radiation-induced defect clusters in fully stabilized zirconia irradiated with ions and/or electrons', J. Nucl. Mater., 319 (2003) 74.

[3] K. Shiiyama, T. Yamamoto, T. Takahashi, A. Guglielmetti, A. Chartier, K. Yasuda, S. Matsumura, K. Yasunaga, C. Meis, 'Molecular dynamics simulations of oxygen Frenkel pairs in cerium dioxide', Nucl. Instr. and Meth. B 268 (2010) 2980.

[4] K. Yasuda, T. Yamamoto, M. Etoh, S. Kawasoe, S. Matsumura, N. Ishikawa, 'Accumulation of radiation damage and disordering in $\mathrm{MgAl}_{2} \mathrm{O}_{4}$ under swift heavy ion irradiation', Int. J. Mater. Res., 102 (2011) 9.

[5] S. Takaki, K. Yasuda, T. Yamamoto, S. Mastumura, N. Ishikawa, 'Atomic Structure of Ion Tracks In Ceria', Nucl. Instr. and Meth. B 326(2014) 140.

[6] K. Yasuda, M. Etoh, K. Sawada, T. Yamamoto, K. Yasunaga, S. Matsumura and N. Ishikawa, 'Defect formation and accumulation in $\mathrm{CeO}_{2}$ irradiated with swift heavy ions', Nucl. Instr. and Meth. B 314 (2013) 185.

[7] K. Yasuda, T. Yamamoto, S. Seki, K. Shiiyama and S. Matsumura, 'Production and Stability of Radiation-Induced Defects in $\mathrm{MgAl}_{2} \mathrm{O}_{4}$ under Electronic Excitation', Nucl. Instr. and Meth. B 266 (2008) 2834. 


\section{Structure of Defects and Microstructure Evolution in Oxide Ceramics}

- Role of Electronic Excitation and Selective Displacement Damage -

\section{Kazuhiro Yasuda}

Department of Applied Quantum Physics and Nuclear Engineering, Kyushu University, JAPAN

MINOS $2^{\text {nd }}$ Int. Workshop Irradiation of Nuclear Materials: Flux and Dose Effects 


\section{Oxide ceramics: fluorite- and spinel-type}

\section{$\square$ Excellent radiation resistance}

- Resistance to amorphization and volumetric swelling

- Successful achievements as LWR fuels: $\mathrm{UO}_{2},(\mathrm{U}, \mathrm{Pu}) \mathrm{O}_{2}$

- Potential applications to inert fuel matrix, transmutation target: stabilized $\mathrm{ZrO}_{2}$

- A surrogate of $\mathrm{UO}_{2}: \mathrm{CeO}_{2}$

\section{$\square$ Radiation effects and radiation resistance}

-Production rate of point defects

$\Rightarrow$ difference between cations and anions

-Recombination rate of point defects

$\Rightarrow$ structural vacancy: cation site (spinel), anion site (YSZ)

-Stability of extended defects: size of stable nuclei (ex. dislocation loop)

-Sensitivity to electronic excitation

$\Rightarrow$ defect kinetics (rather low electronic stopping)

$\Rightarrow$ ion tracks (high electronic stopping) 


\section{Topics}

$\square$ Selective displacement damage of oxygen sub-lattice

$\square$ Structure of ion tracks

प Stability of dislocation loops under electronic excitation 


\section{$\square$ High production rate and mobility of oxygen defects} (Fluorite-type)

\begin{tabular}{|c|c|c|c|}
\hline \multicolumn{1}{|c|}{$\mathrm{CeO}_{2}$} & $\begin{array}{c}\mathrm{Ce} \text { ion } \\
\text { (cation) }\end{array}$ & $\begin{array}{c}\mathrm{O} \text { ion } \\
\text { (anion) }\end{array}$ & Ref. \\
\hline mass [amu] & 140 & 16 & \\
\hline Displacemend energy [eV] & $44 \sim 58$ & $<30$ & {$[1]$} \\
\hline $\mathrm{V}$ - migration enegy [eV] & $2.1 \sim 5.4$ & $0.5 \sim 0.6$ & {$[1],[2]$} \\
\hline 1- migration energy [eV] & 6.1 & 1.1 & {$[2]$} \\
\hline
\end{tabular}

[1] K. Yasunaga, et al, NIMB 266 (2008) 2877. [2] A. Guglielmetti et al, NIMB (2008) 5120.

$\Rightarrow$ Role of oxygen point defects is important for defect kinetics in fluorite-type oxides. 


\section{Anomalous defects in YSZ: selective displacement of O-ions}

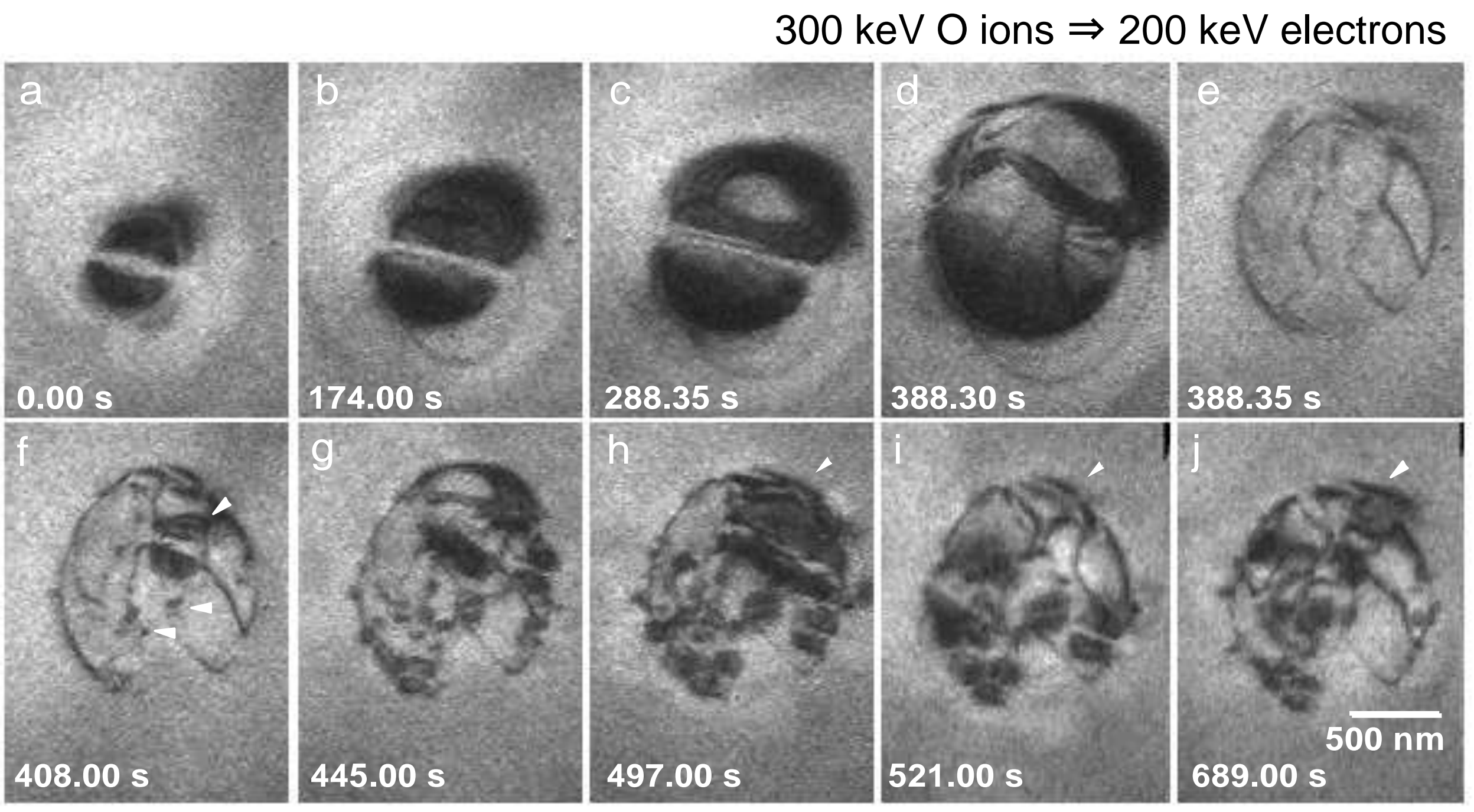

K Yasuda et al, JNM 319 (2003) 74. 
- Strong stress and strain field around the defect

- Entirely different growth process

- Multiplication of dislocations during the growth.

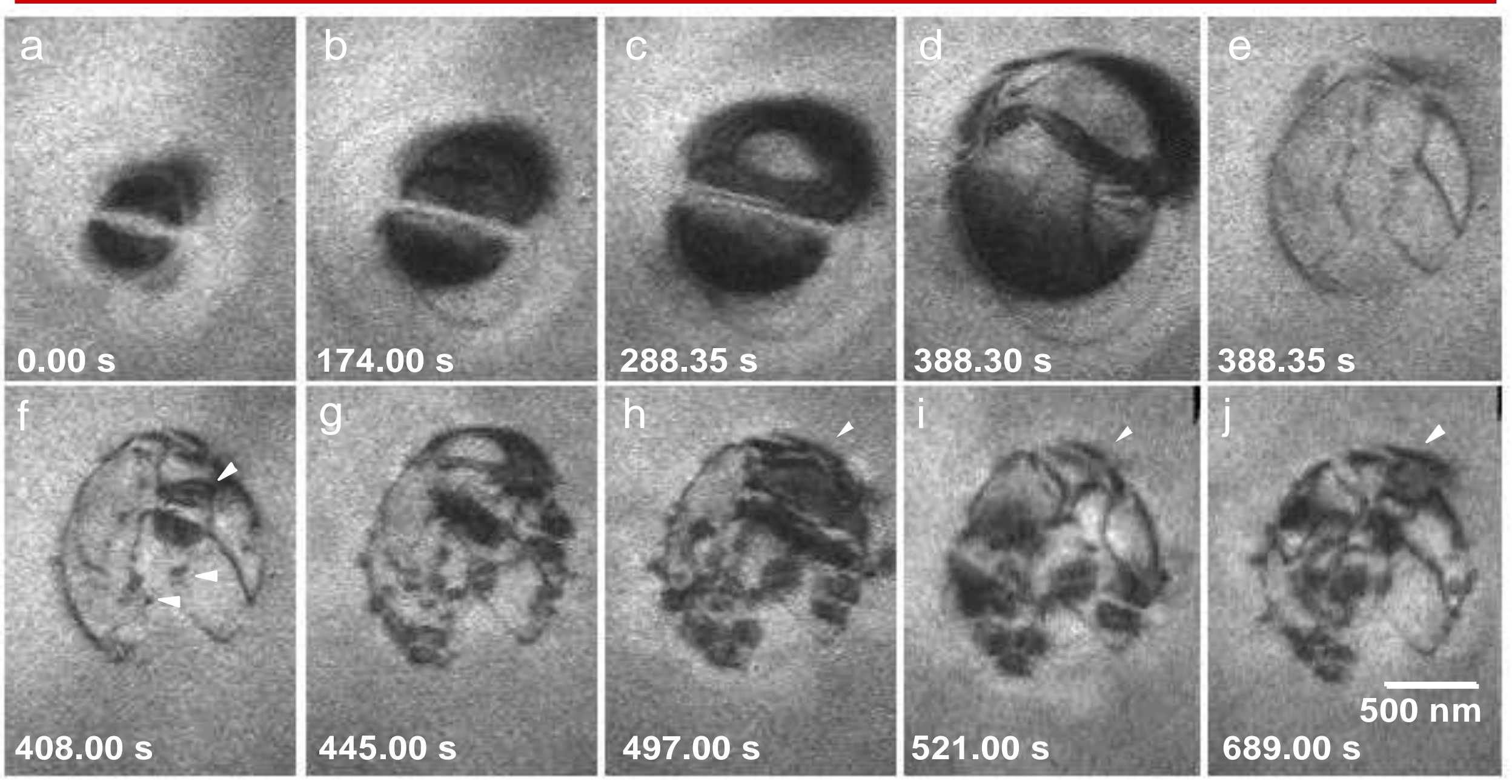




\section{Similar oxygen-type defects (loops) in $\mathrm{CeO}_{2}$}

(at $300 \mathrm{~K} . \Phi \sim 3 \times 10^{26} \mathrm{e} / \mathrm{m}^{2}$ )

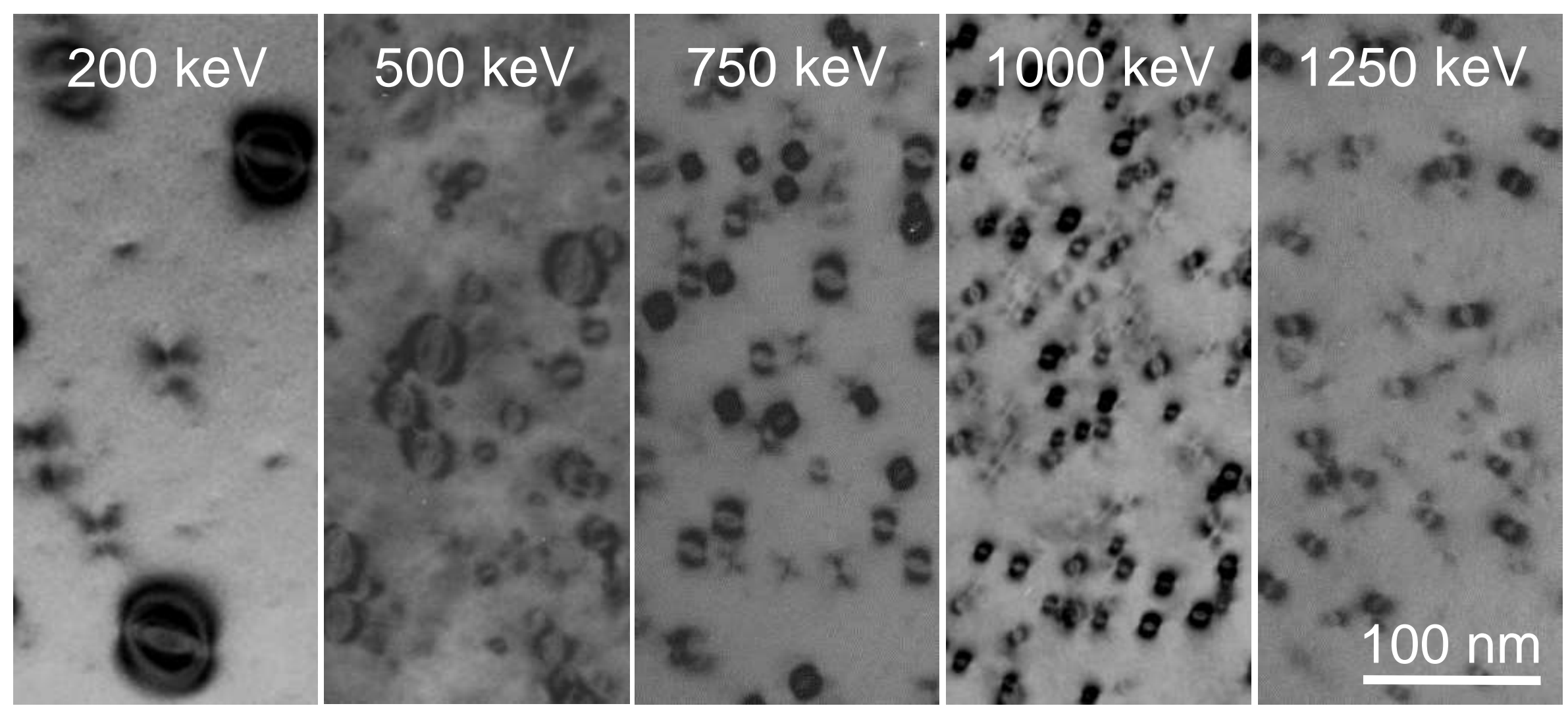

With decreasing e-energy, loop size is increased and density is decreased. $\vec{B} / /<111>$, on (111) planes K Yasunaga et al, NIMB (2008) 


\section{A model for charged disl. loop consist of O-ions}

A. Ryazanov et al, JNM 323 (2003) 372.

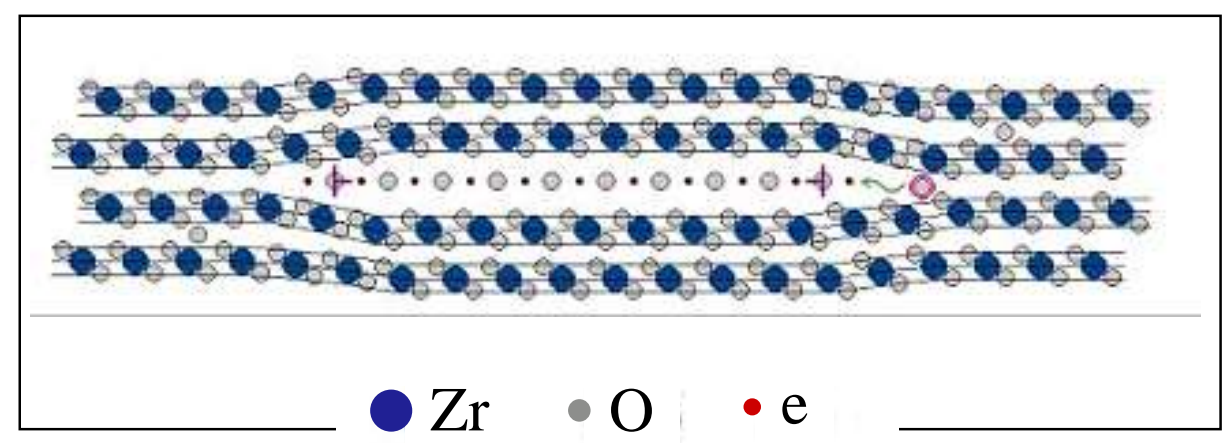

$$
\begin{gathered}
\sigma_{i k}=\sigma_{i k}^{E}+\sigma_{i k}^{Y} \\
\begin{array}{l}
\text { driven by an } \\
\text { electric field }
\end{array} \quad \begin{array}{l}
\text { driven by an } \\
\text { elastic strain }
\end{array}
\end{gathered}
$$

$\square$ accumulation of oxygen ions, preferentially at dislocations or invisible defect clusters

$\square$ oxygen ions are considered to lose electrons during diffusion process

$\square$ the defect clusters are considered to trap free electrons and grow as a charged dislocation loop

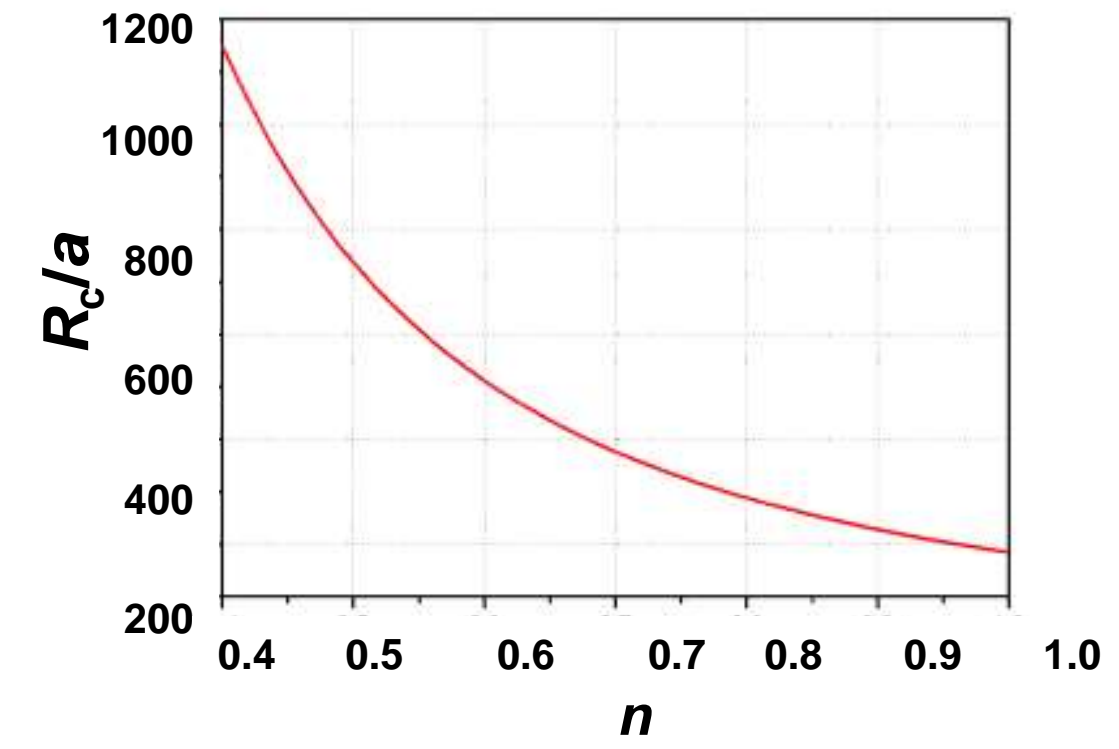

Charge of $\mathrm{O}^{-n}$ ions 
HAADF STEM image of Dislocation loops in $\mathrm{CeO}_{2}$

$>200 \mathrm{keV}$ electron irradiation at $300 \mathrm{~K}$
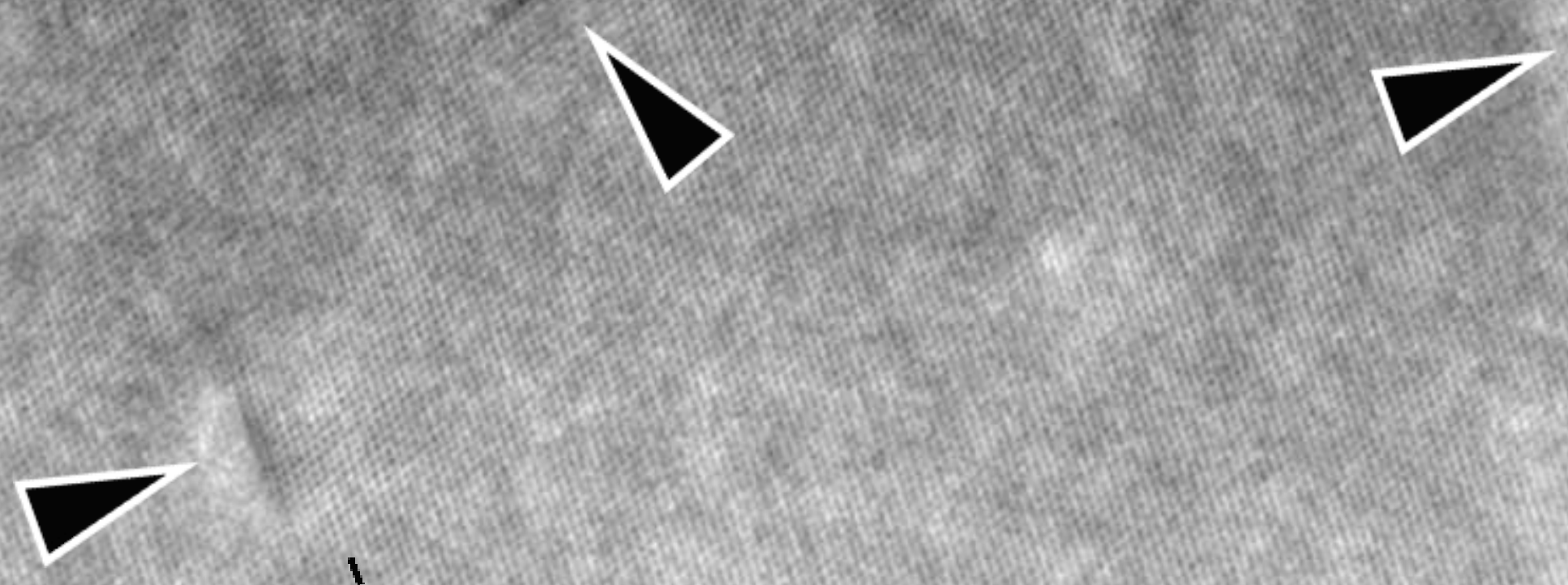

I

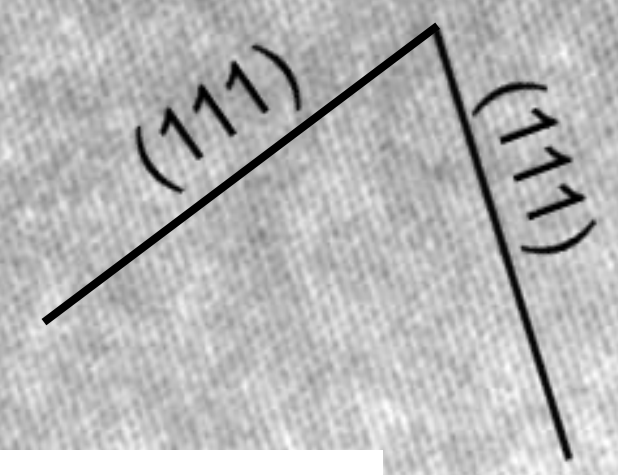

$10 \mathrm{~nm}$

S. Takaki et al. Mater. Res. Soc. Symp. Proc. 1514 (2013) 93.

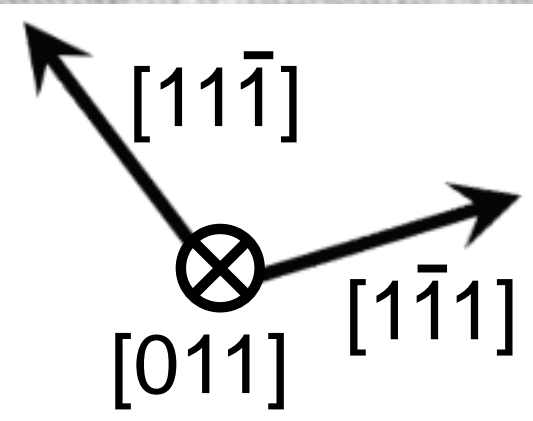




\section{HAADF STEM image}

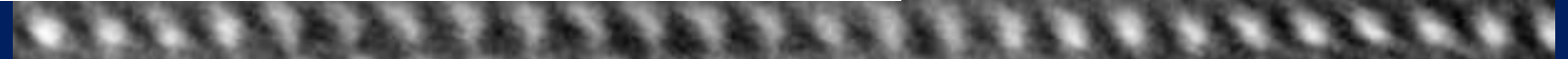

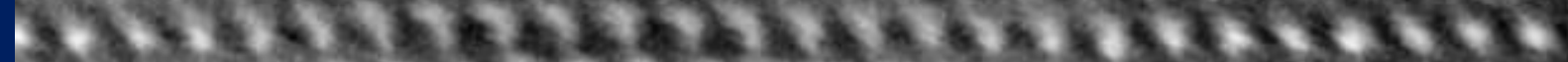

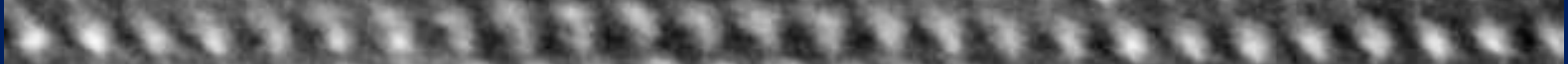

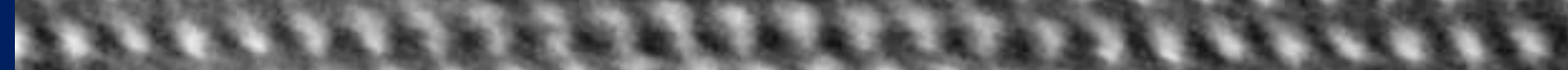

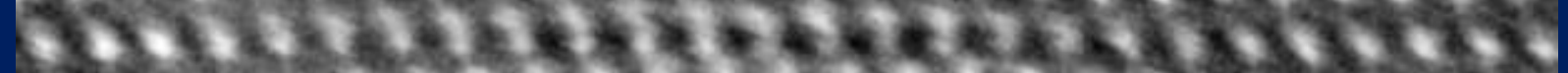

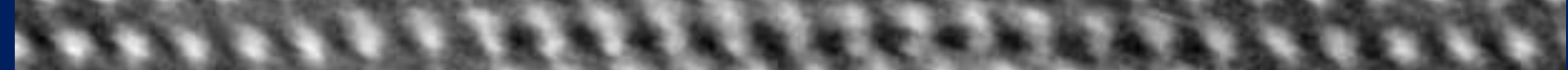

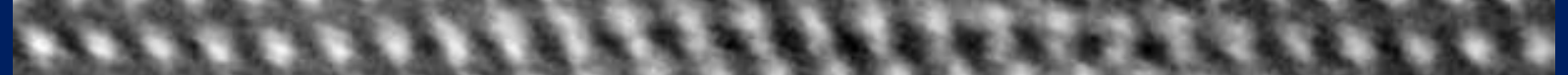

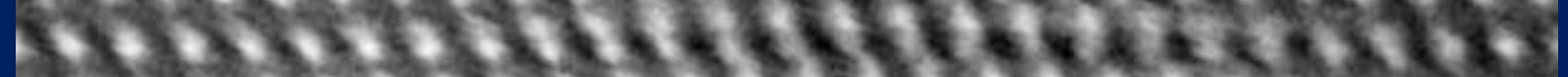

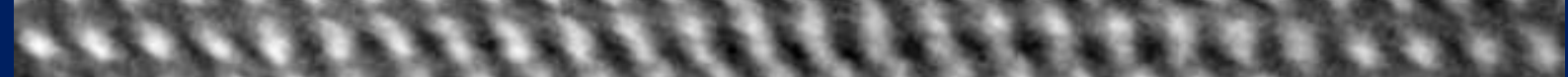

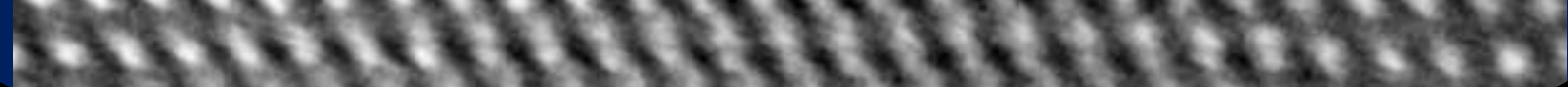

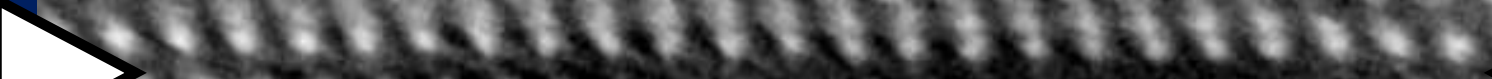

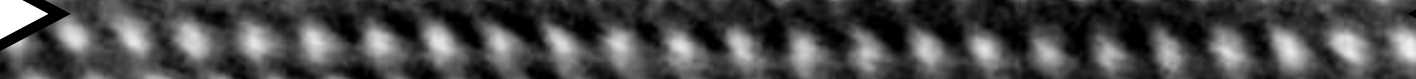

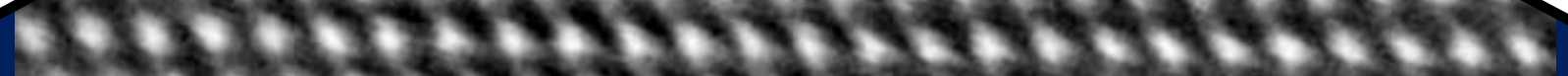

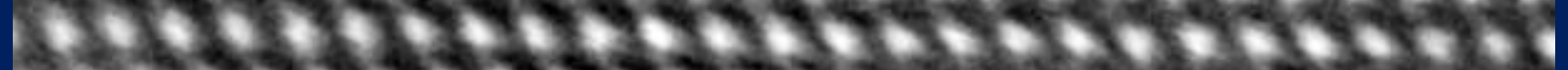

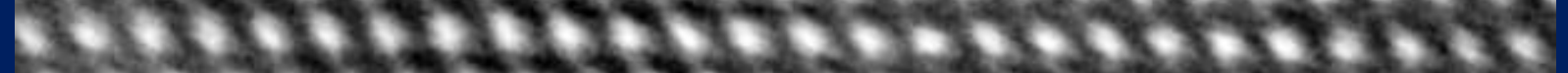
CW

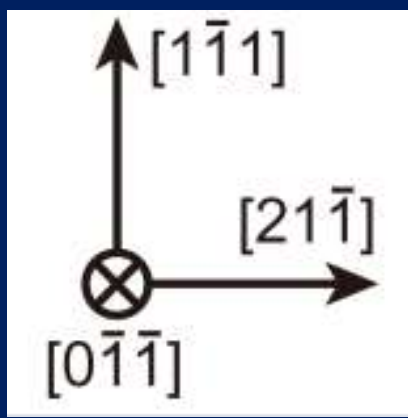

Ce ion

C O ion

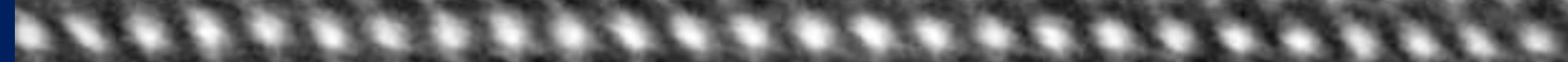
1.

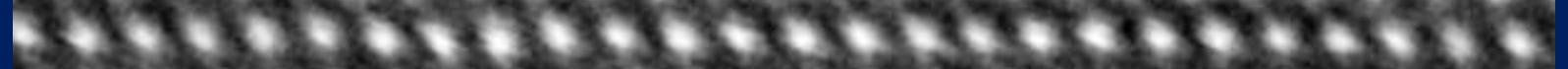

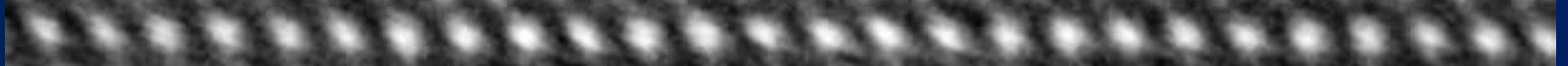

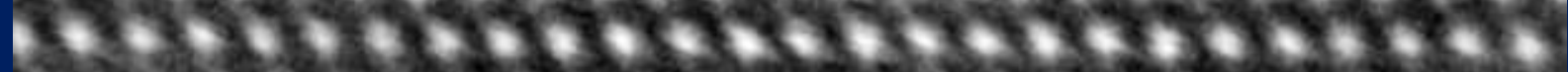

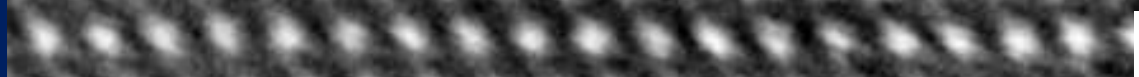

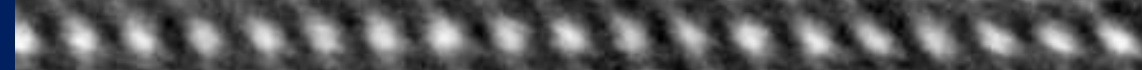

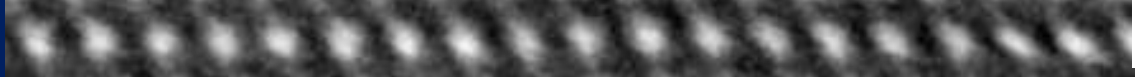

\section{$2 \mathrm{~nm}$}

- Lattice planes are strongly distorted around the dislocation loop.

- No additional Ce-plane is inserted at the dislocation loop, indicating that this is not the perfect dislocation loop.

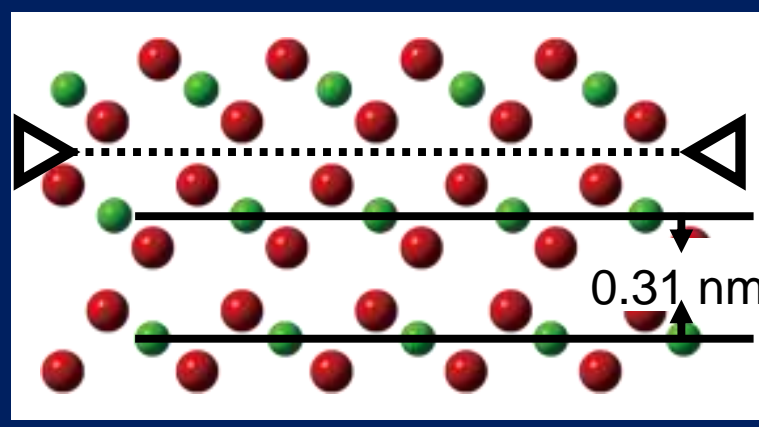



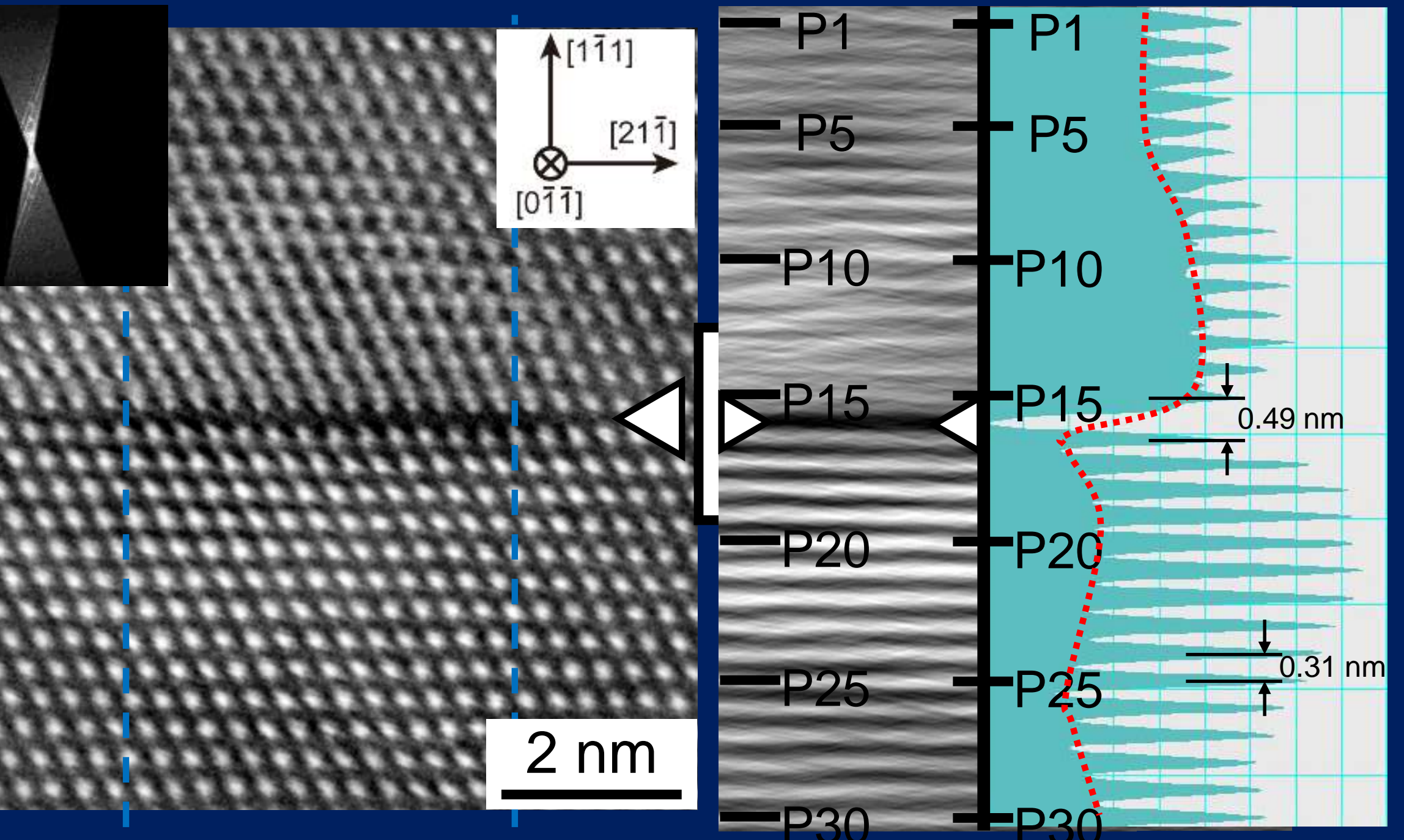

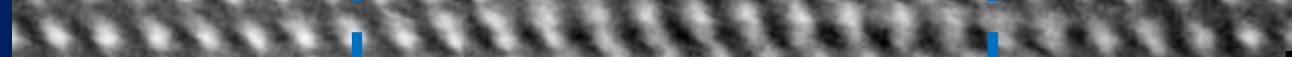

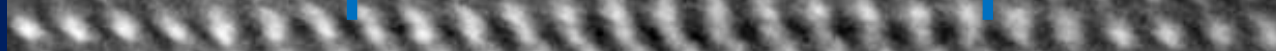

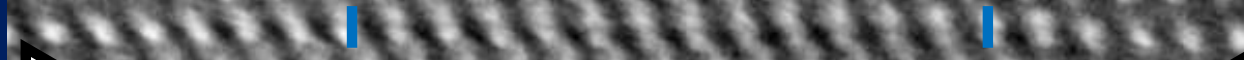

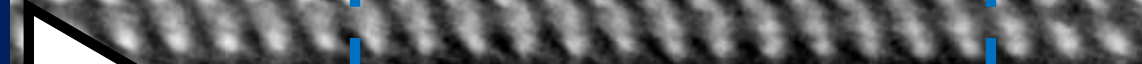

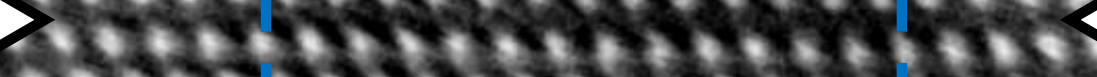

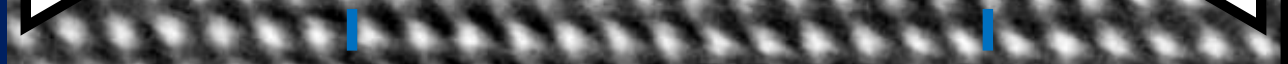

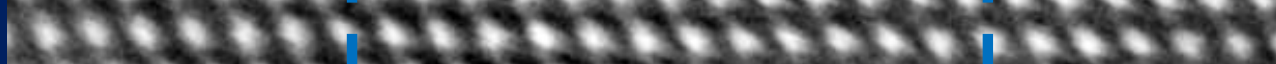

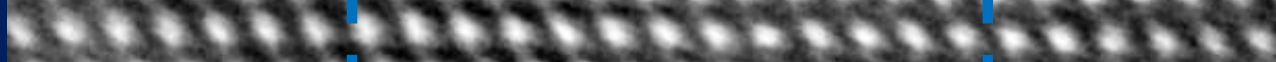

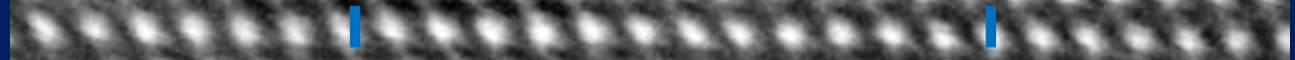

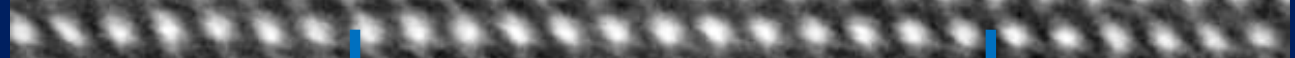

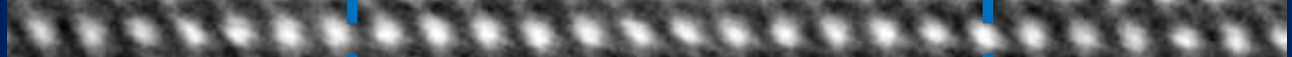

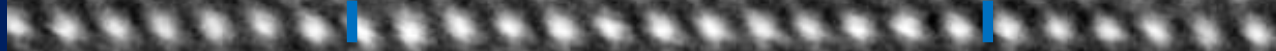

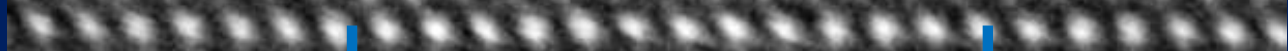

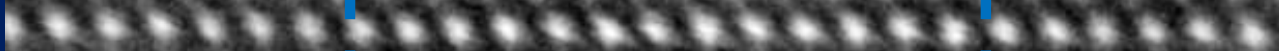

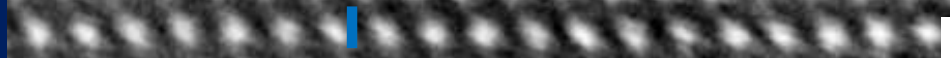

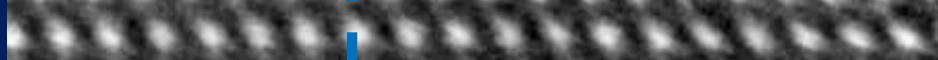
cos.

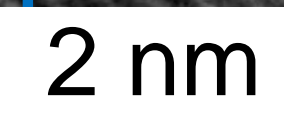

$\Rightarrow$ The loop is suggested to be on a (111) plane consist of oxygen ions. 


\section{Topics}

$\square$ Selective displacement damage of oxygen sub-lattice

口 Structure of ion tracks

$\square$ Stability of dislocation loops under electronic excitation 
口 Fission fragments $(E \sim 70-100 \mathrm{MeV})$

- high-density electronic excitation $\left(\mathrm{S}_{\mathrm{e}} \sim 20 \mathrm{keV} / \mathrm{nm}\right)$

- ion tracks

swift heavy ion

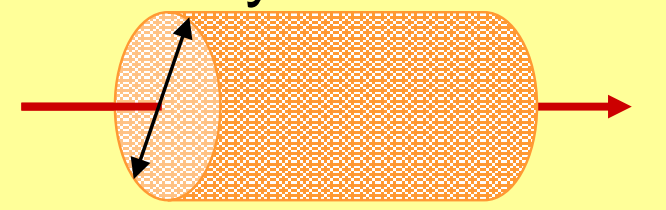

ion track

\section{$\square$ lon tracks in fluorite and spinel-type oxides}

- radiation resistant: no amorphization by individual ions

- threshold $S_{e}$ for continuous track formation

$\sim 10 \mathrm{keV} / \mathrm{nm}: \mathrm{MgAl}_{2} \mathrm{O}_{4}, \sim 15 \mathrm{keV} / \mathrm{nm}: \mathrm{CeO}_{2}$

- At high fluence $\Rightarrow$ dislocation structure

$\mathrm{MgAl}_{2} \mathrm{O}_{4}$ : amorphization $\left(10^{20} \mathrm{~m}^{-2}\right)$

(Zinkle 2000)

$\mathrm{CeO}_{2}$ : sub-grain formation

(Sonoda 2010, Garrido 2009)

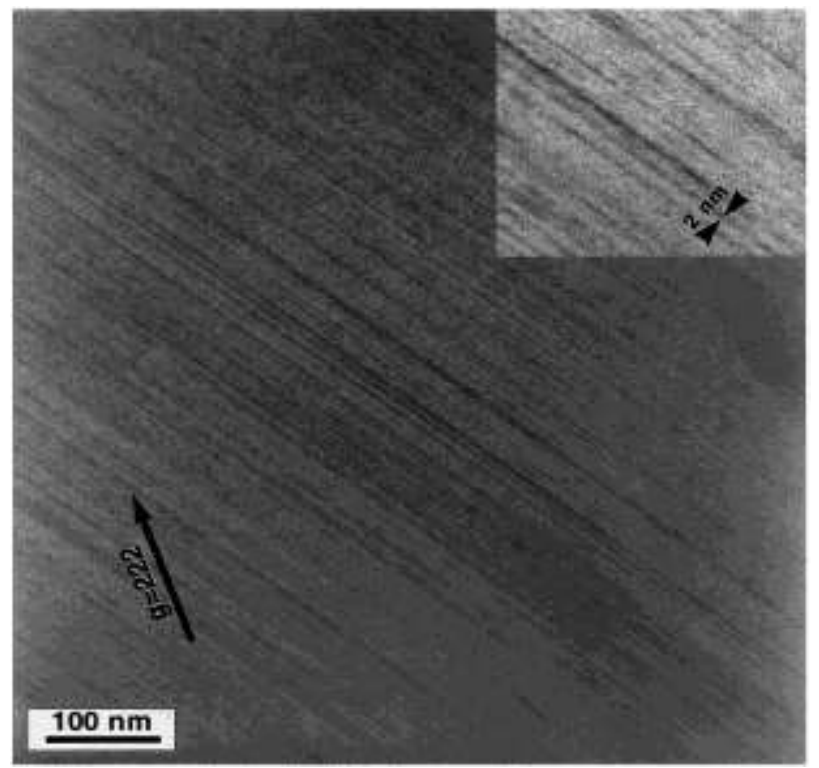

S.J. Zinkle et al., NIMB 141 (1998) 


\section{$\mathrm{CeO}_{2}$ irradiated with $200 \mathrm{MeV}$ Xe : $S_{e}=27 \mathrm{keV} / \mathrm{nm}$}

\section{Bright-field kinematical TEM images}

$3 \times 10^{11}\left(\mathrm{~cm}^{-2}\right)$

(a) Under-focus

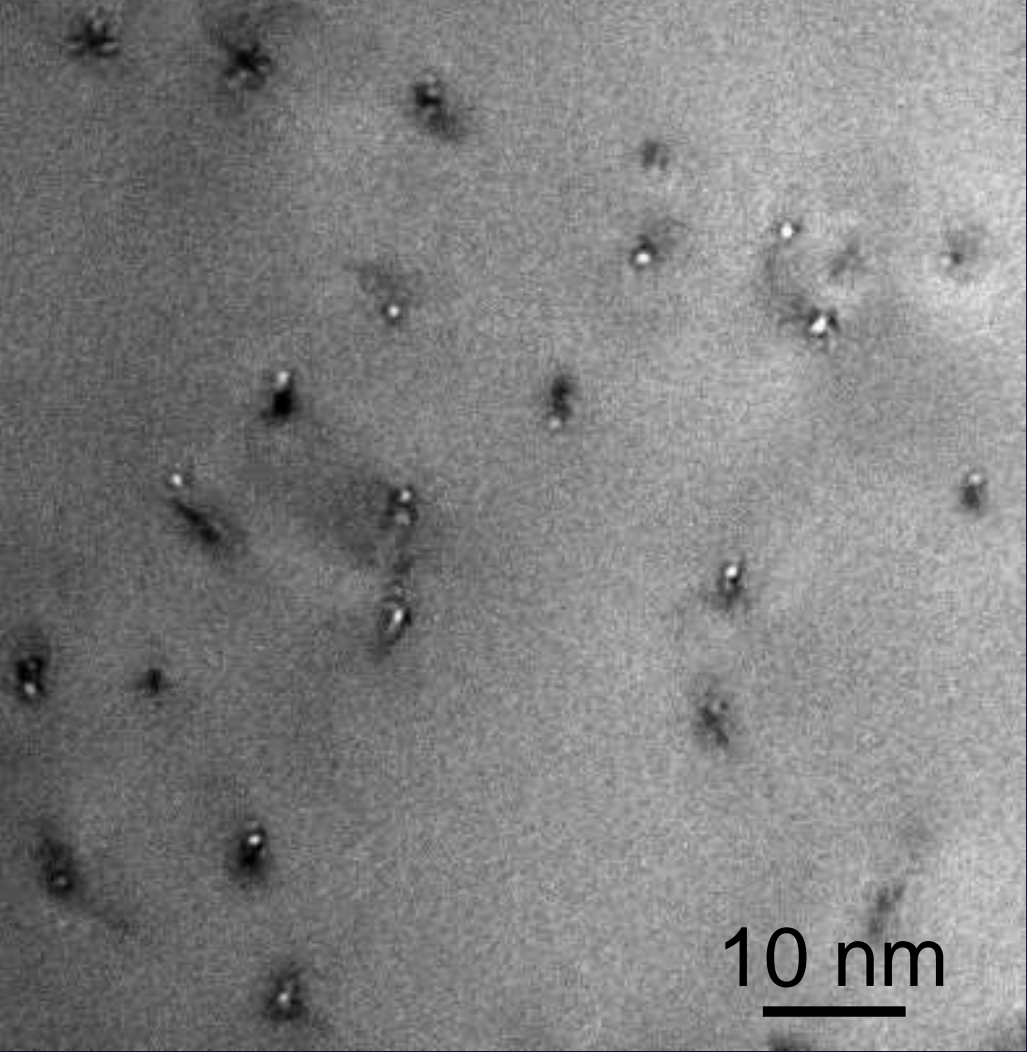

(b) Over-focus
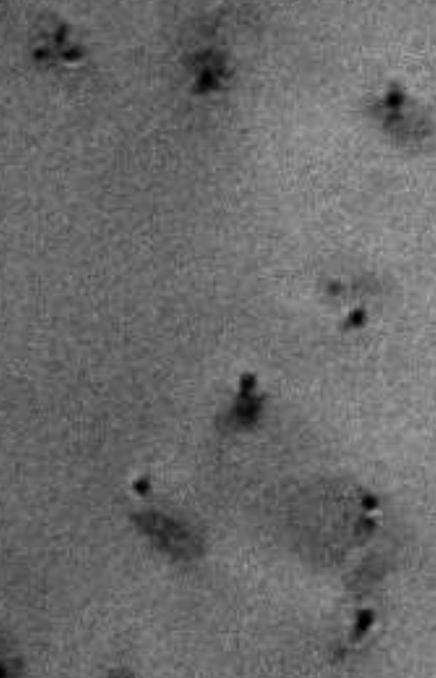

8.
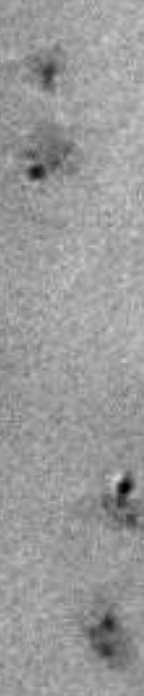

2.

- Ion tracks appear as Fresnel contrast $\Rightarrow$ decrease in atomic density. - Fluorite structure is retained. 


\section{$\mathrm{MgAl}_{2} \mathrm{O}_{4}:$ Bright-field TEM images}

\section{kinematical (off-Bragg) diffraction condition $\quad 5 \times 10^{15}\left(\mathrm{~m}^{-2}\right)$}

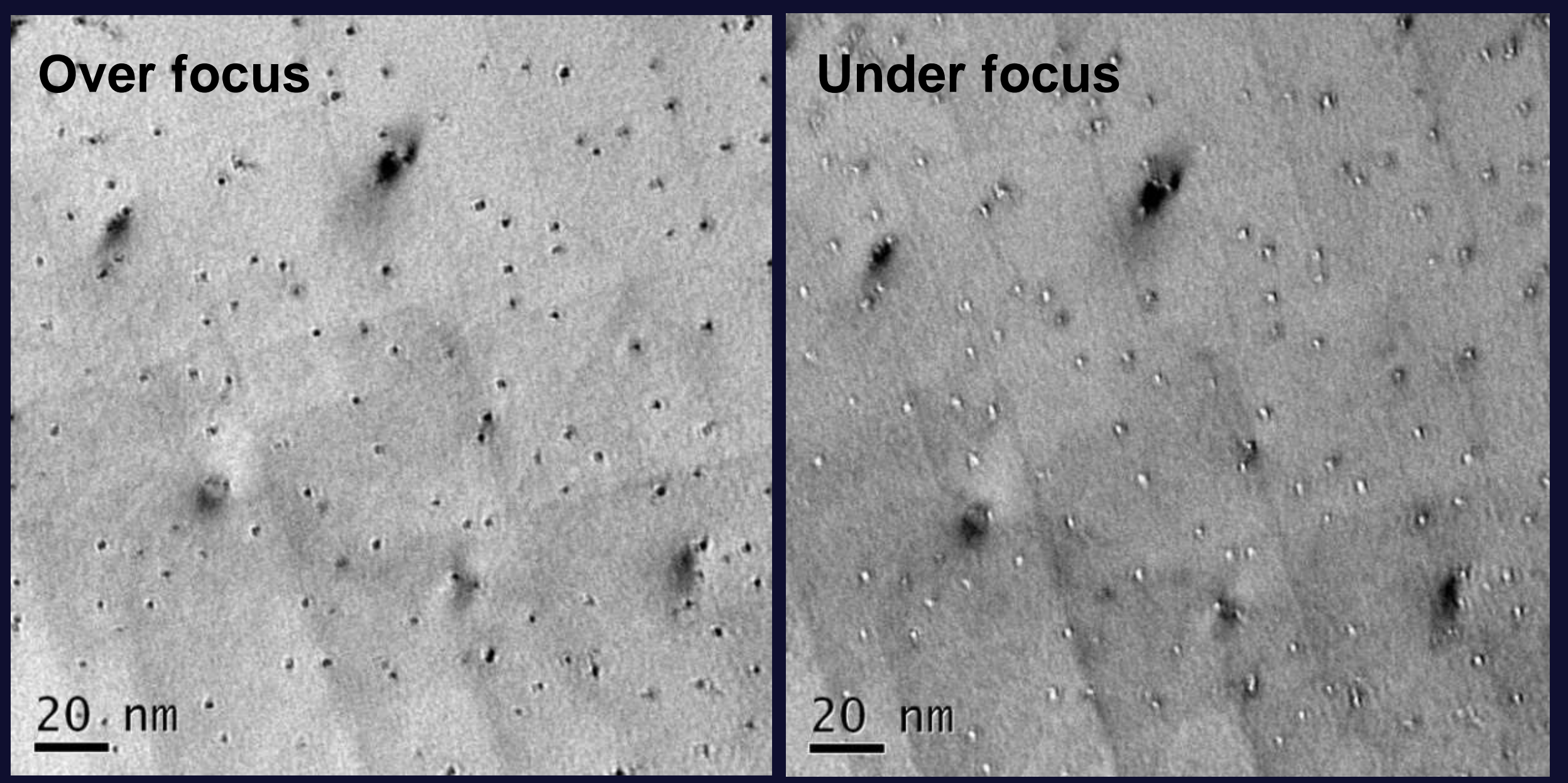

口 Core damage regions (2-3 nm insize) show Fresnel contrast.

K. Yasuda et al. Int. J. Mater. Res. (2011) 140. 
HAADF STEM Image of an Ion Track ::::: $\mathrm{CeO}_{2}$ with $200 \mathrm{MeV} \mathrm{Xe}$

$3 \times 10^{12}\left(\mathrm{~cm}^{-2}\right)$

Es

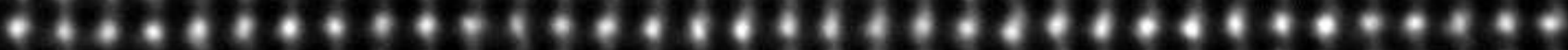

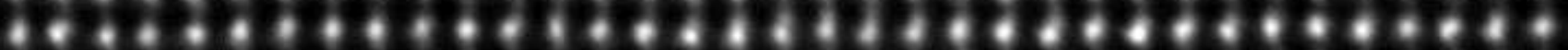

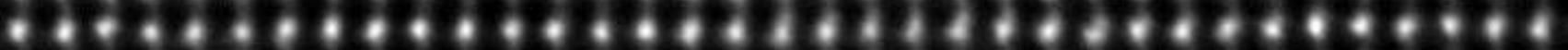

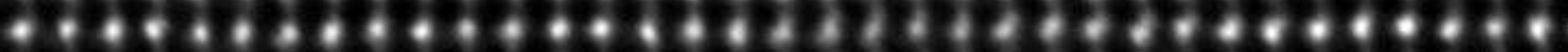

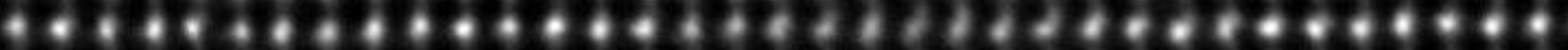

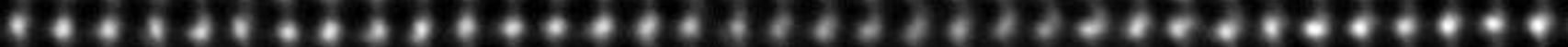

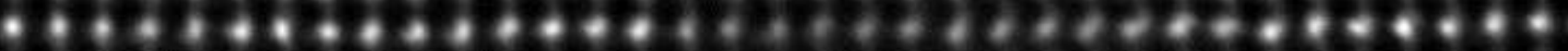

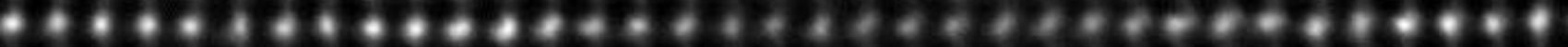

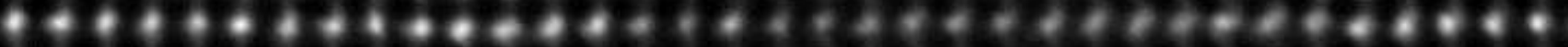

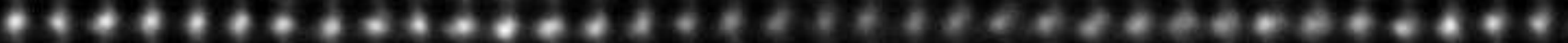

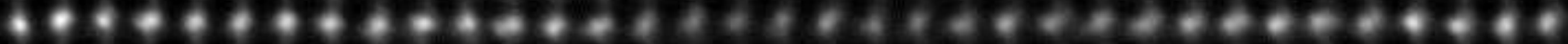

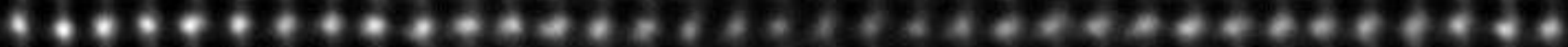

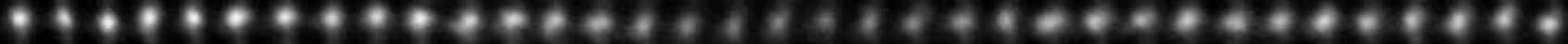

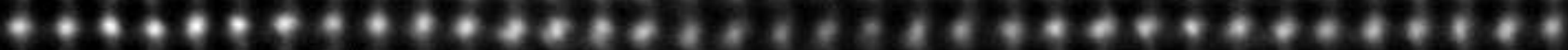

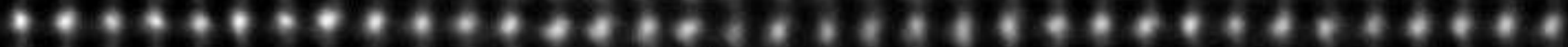

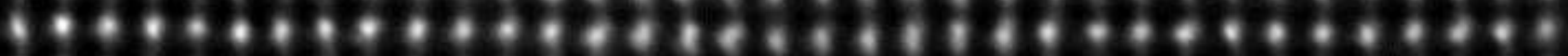

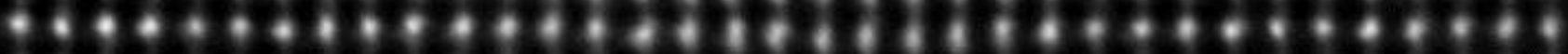

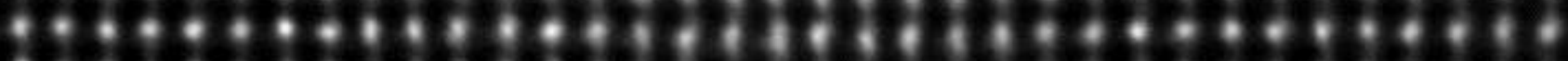

a.0.

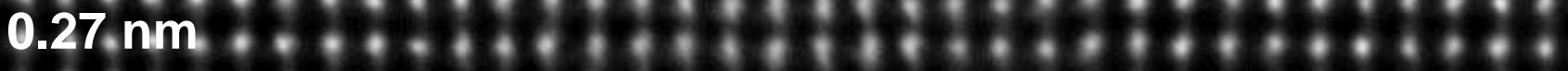

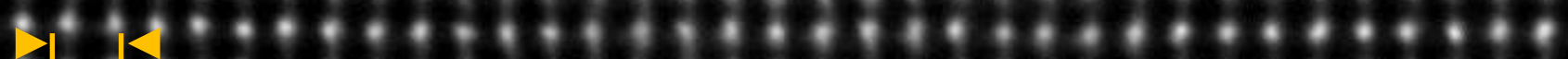

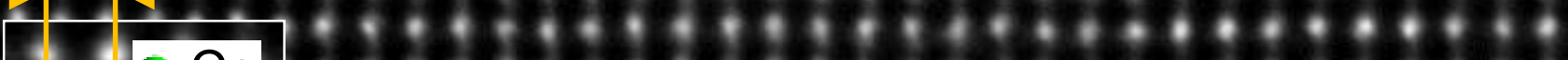

$\begin{array}{lll}0 & \mathrm{Ce} \\ \mathrm{O} & \mathrm{O}\end{array}$

$0.27 \mathrm{~nm}$

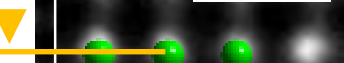

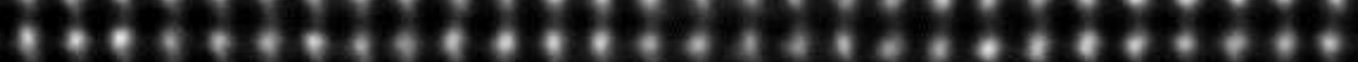

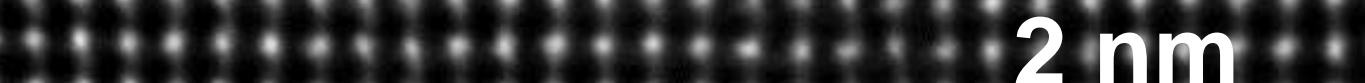

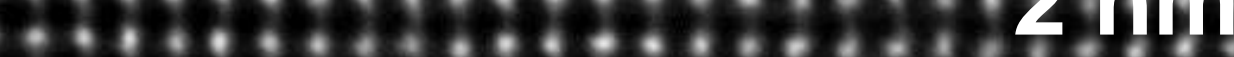

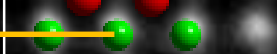

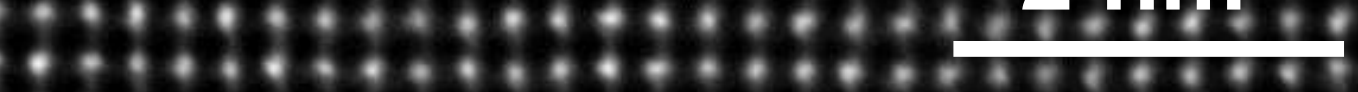

N/y KYUSHU UNIVERSITY

S. Takaki et al. NIMB 326 (2014) 140. 
ABF STEM Image of an Ion Track (1) Q.6.

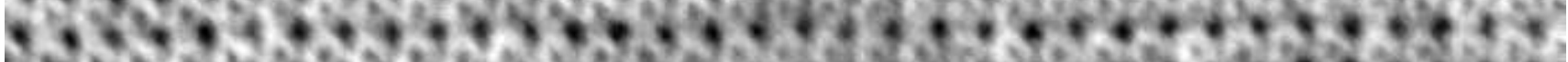

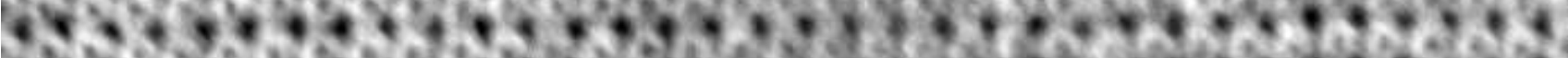
1.3.

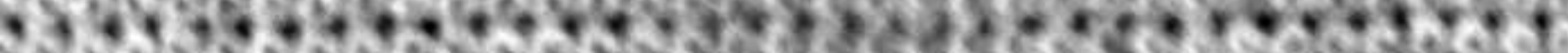

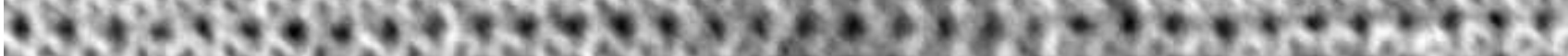
9.8. 6.8. Q.3.

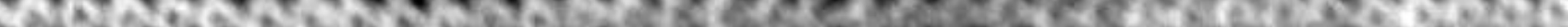
Q.

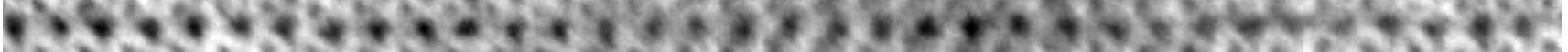

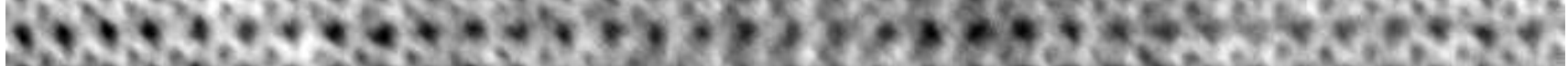

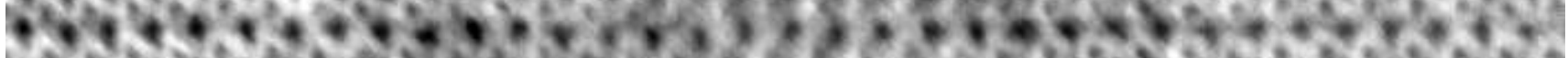

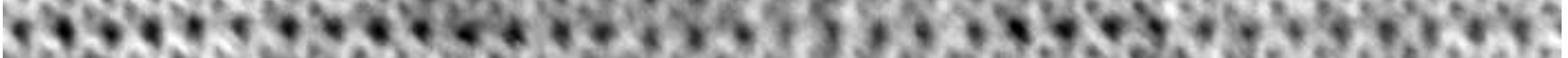
S.M.

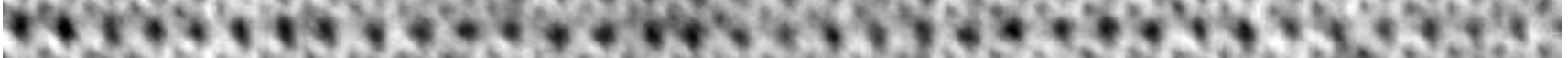

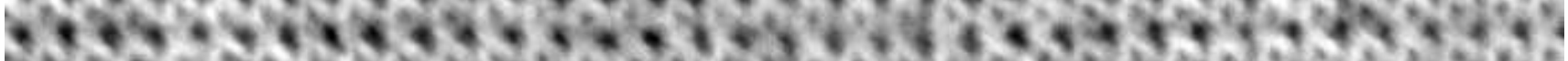

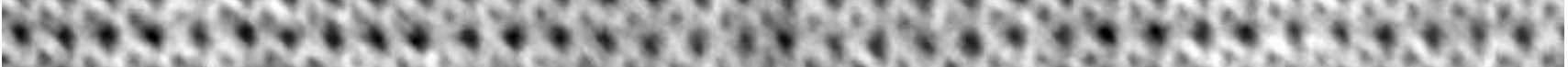

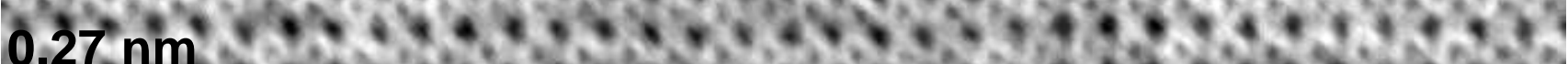

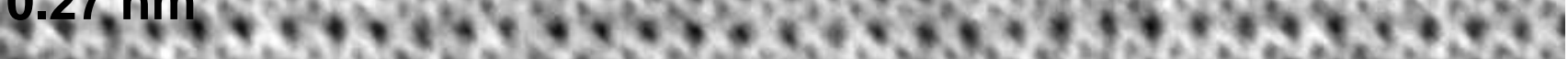

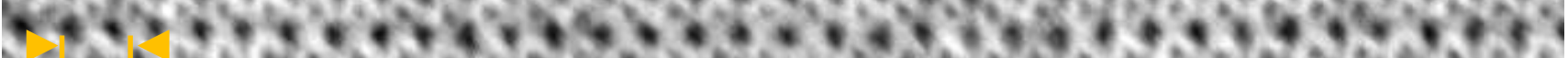
o Ce

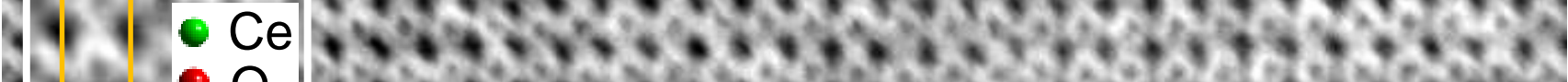

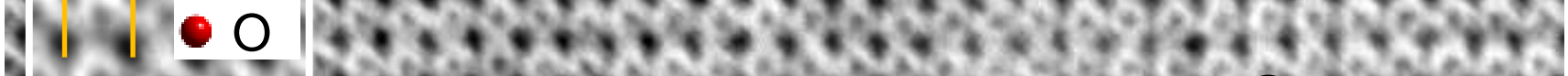
VIr. $0.27 \mathrm{~nm}$

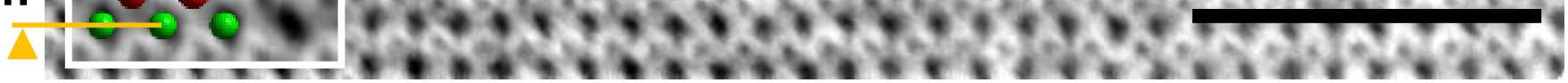

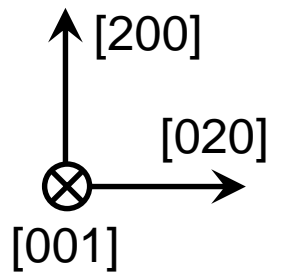



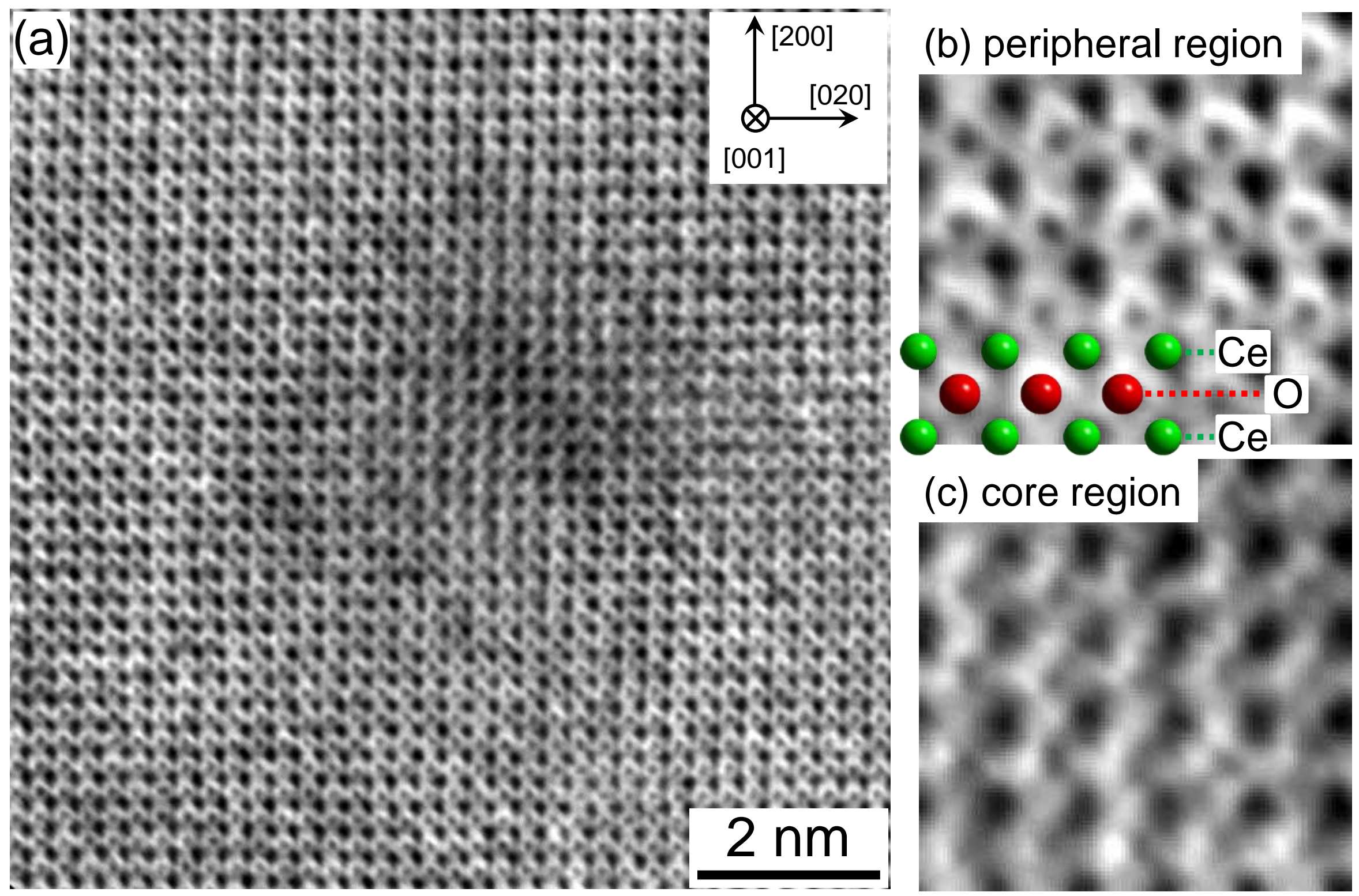

N/y KYUSHU UNIVERSITY 


\section{Accumulation of core damage region}

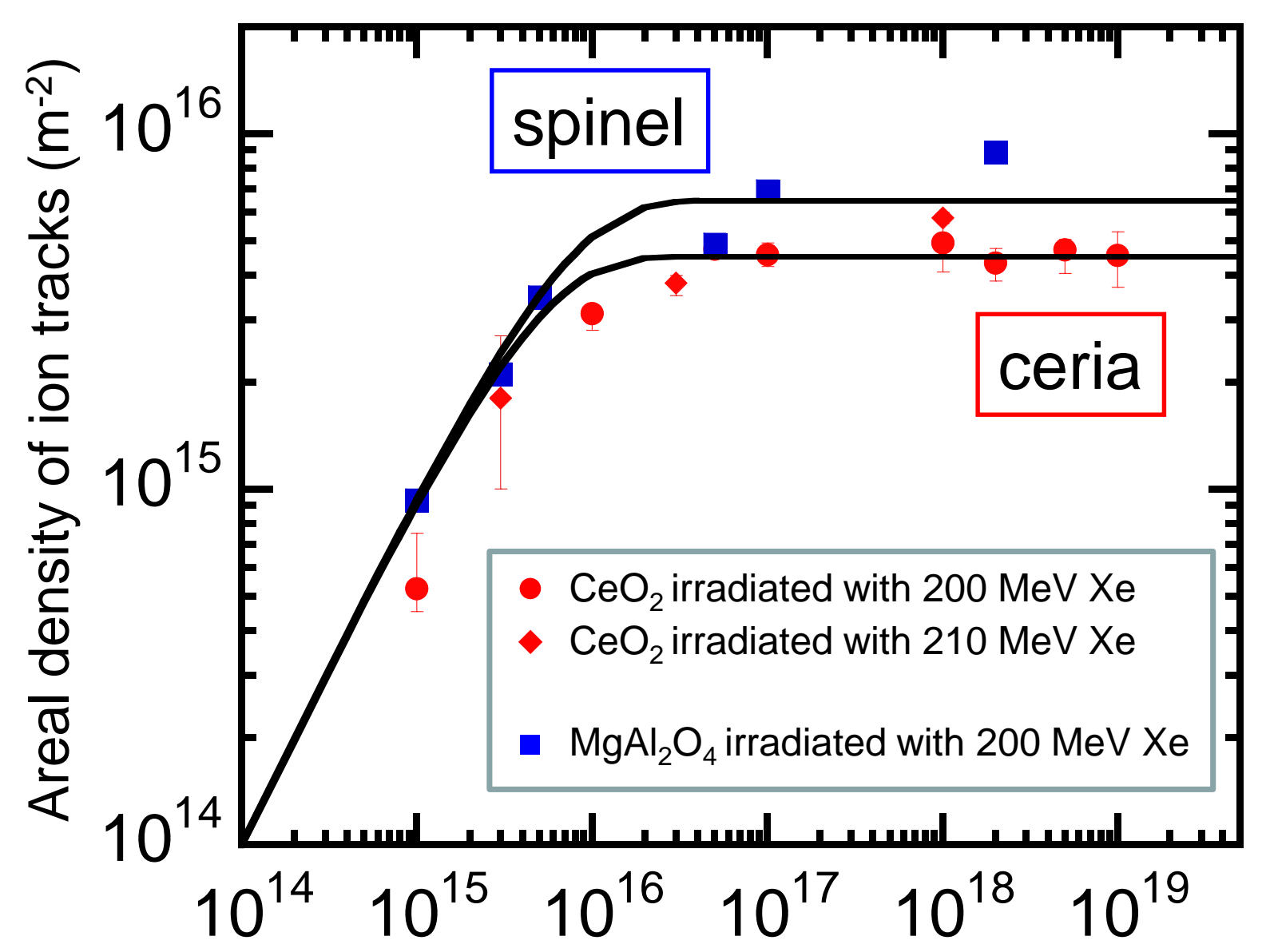

BF-TEM Fresnel contrast

(a) Under-focus

Fluence (ions $/ \mathrm{m}^{2}$ )

$\square$ The density is saturated at high fluence, although damage area does not covers the whole region. $\Rightarrow$ balance between the production and recovery 


\section{Accumulation of core damage region}

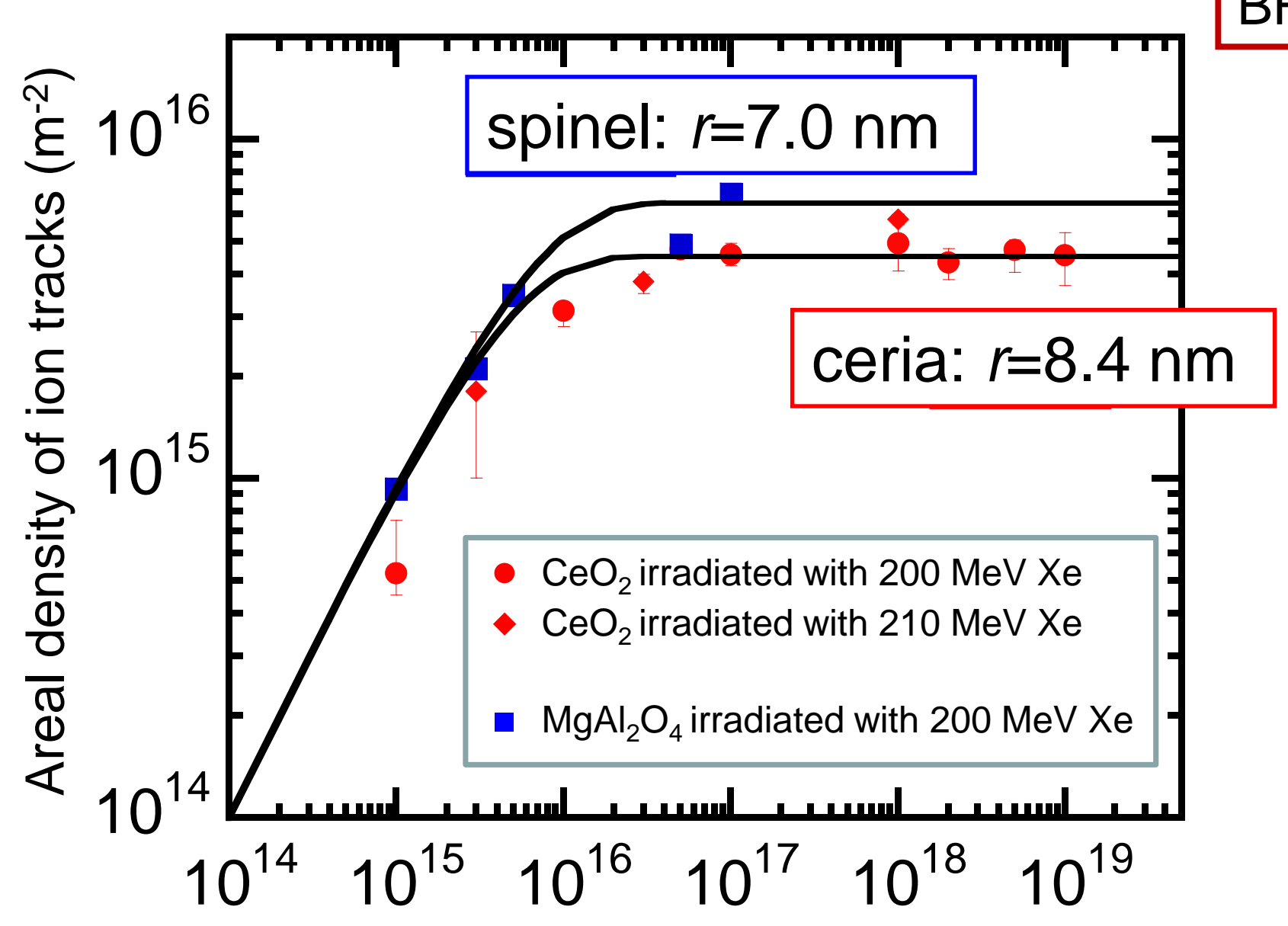

BF-TEM Fresnel contrast

pre-existing core damage region

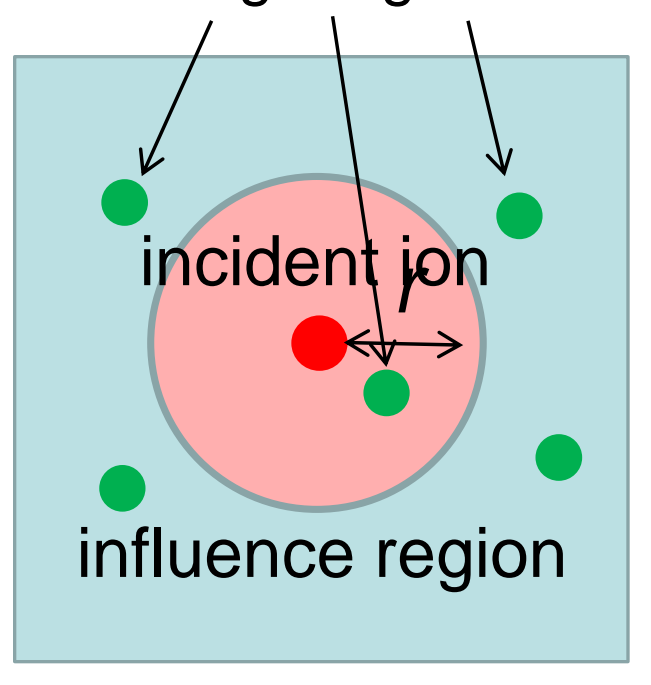

Fluence (ions $\left./ \mathrm{m}^{2}\right)$

- Interstitials are generated during the recovery process in the influence region to create core damage regions with high concentration of vacancy. 


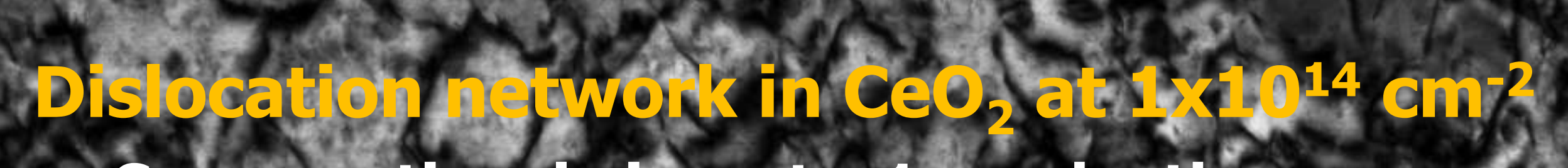
cross sectional visw at $1_{1} \mu \mathrm{m}$ depth 1 ,

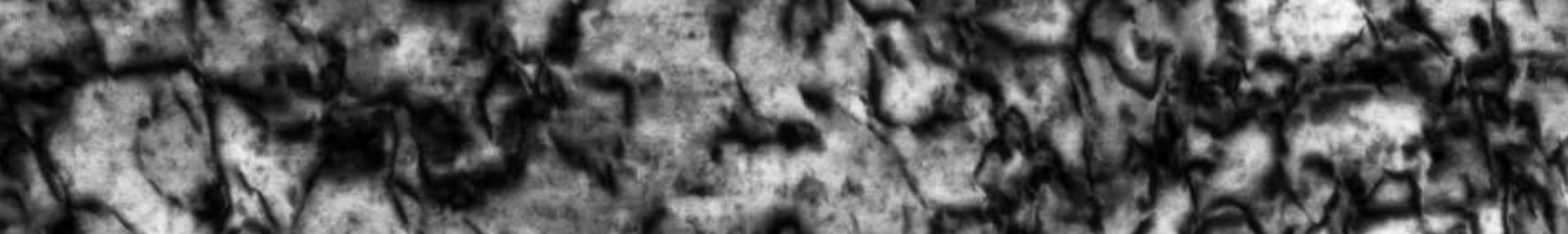

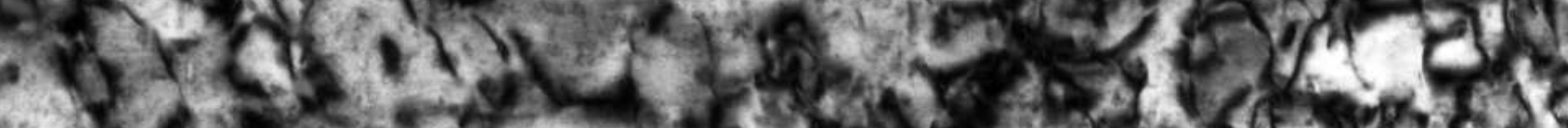

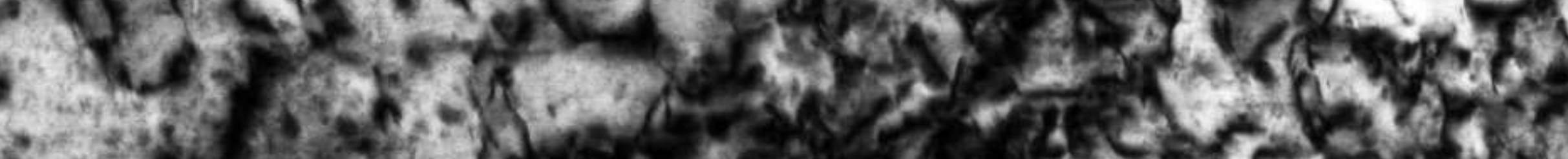

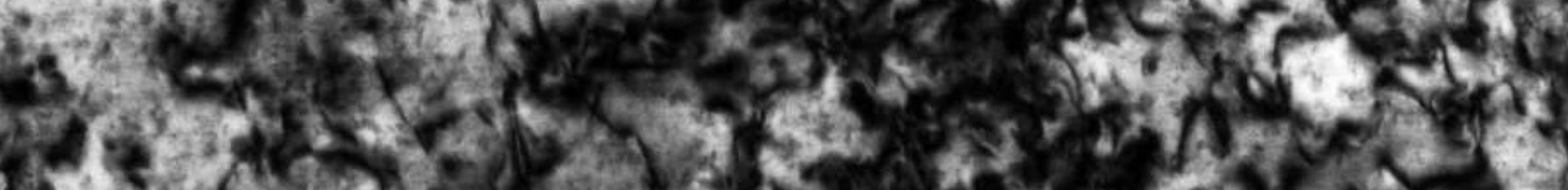

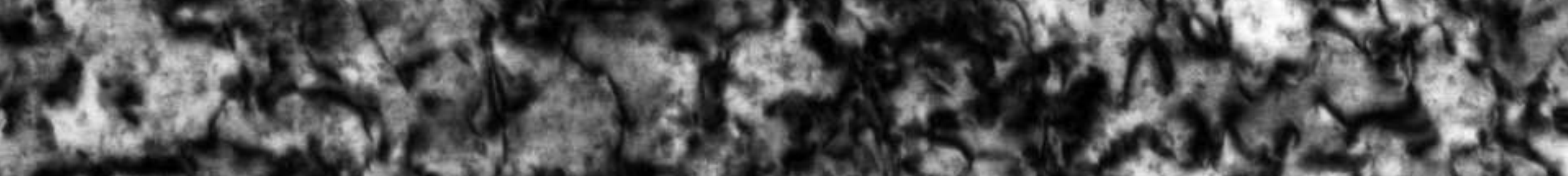
Ha 


\section{Formation of subgrains at near surface region}

$210 \mathrm{MeV}$ Xe ions: $1 \times 10^{16}$ ions $/ \mathrm{cm}^{2}$ at $573 \mathrm{~K} \Rightarrow$ overlap with $\sim 10^{4}$ times

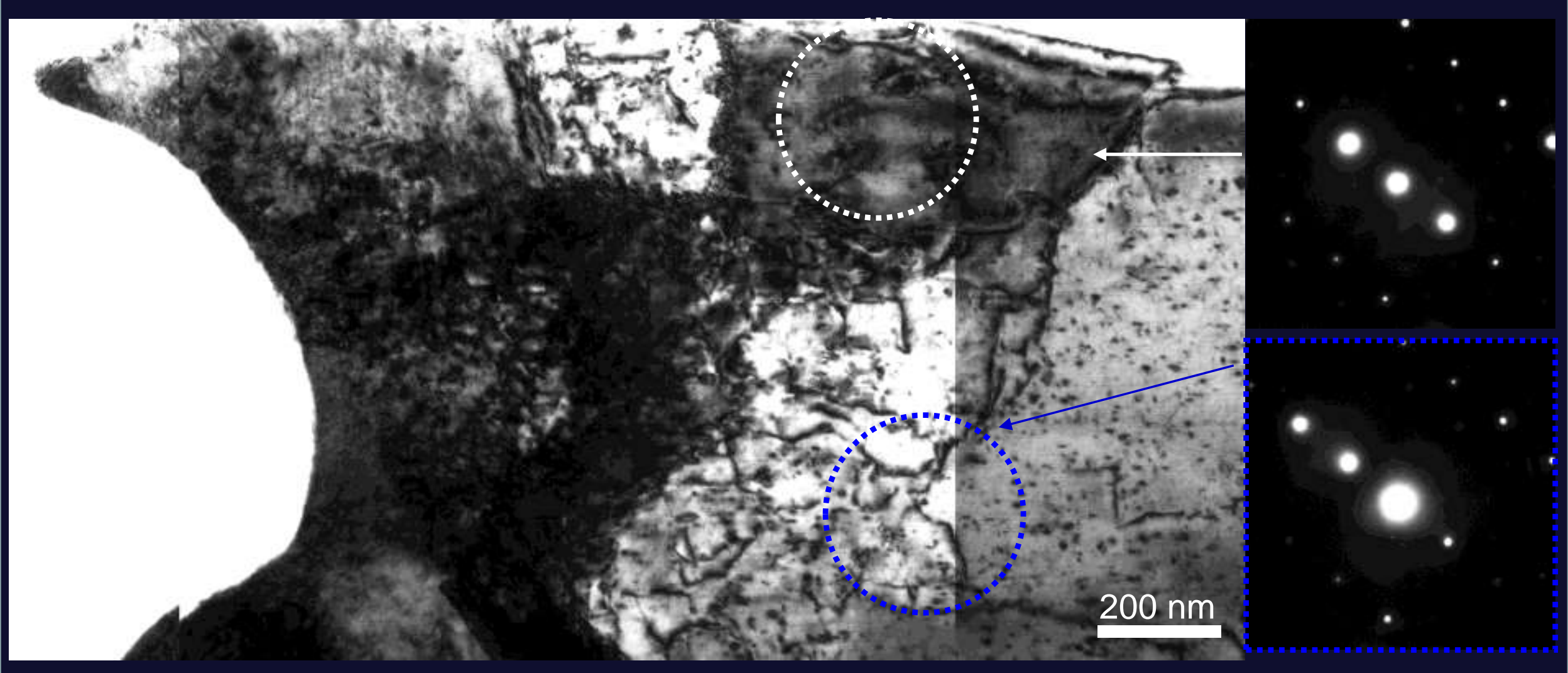




\section{Topics}

$\square$ Selective displacement damage of oxygen sub-lattice

$\square$ Structure of ion tracks

- Stability of dislocation loops under electronic excitation 


\section{$\square$ Displacement damage and electronic excitation}

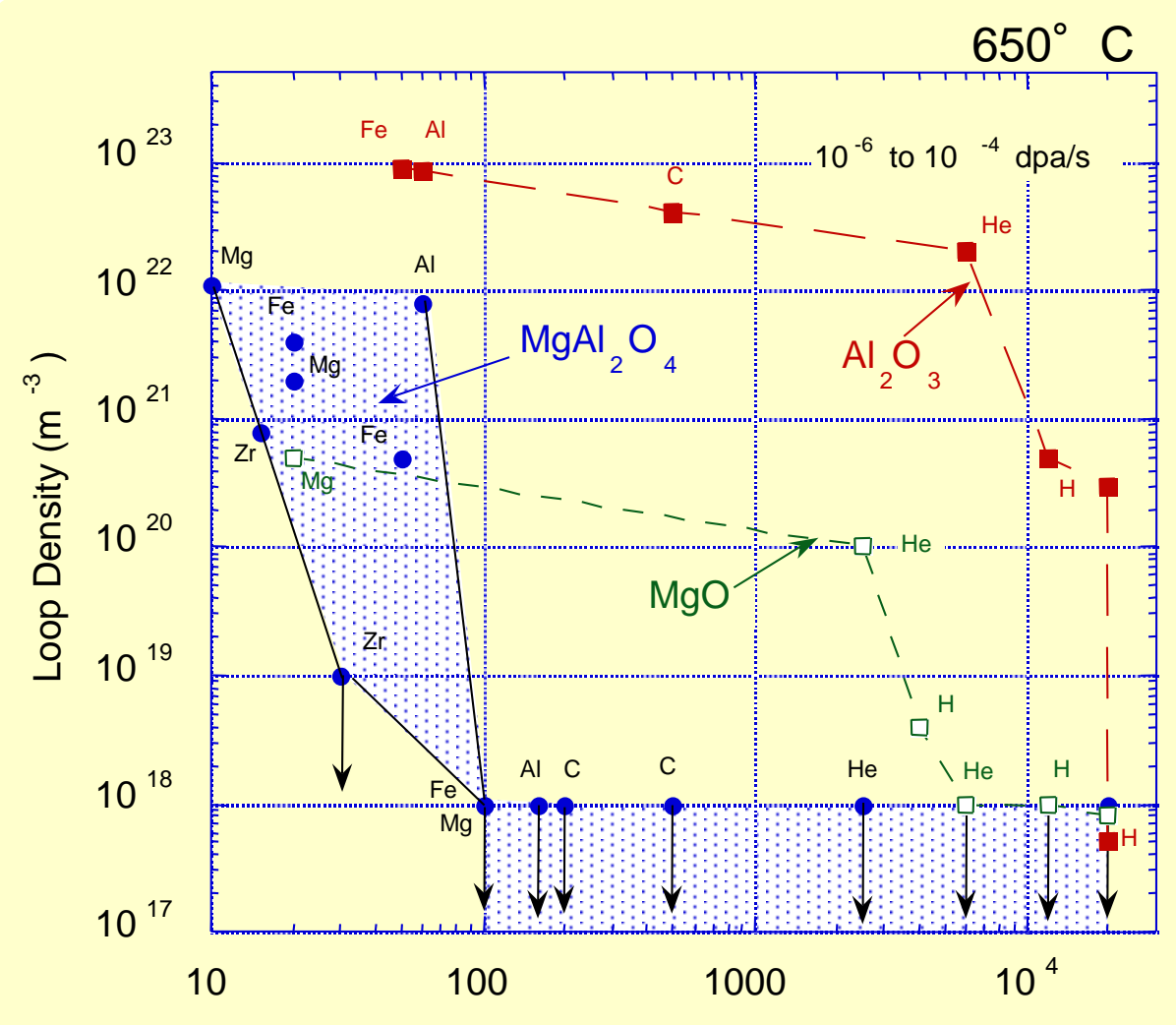

Electron-hole pairs/dpa Ratio

S.J. Zinkle, MRS Symp. Proc. 439 (1997)

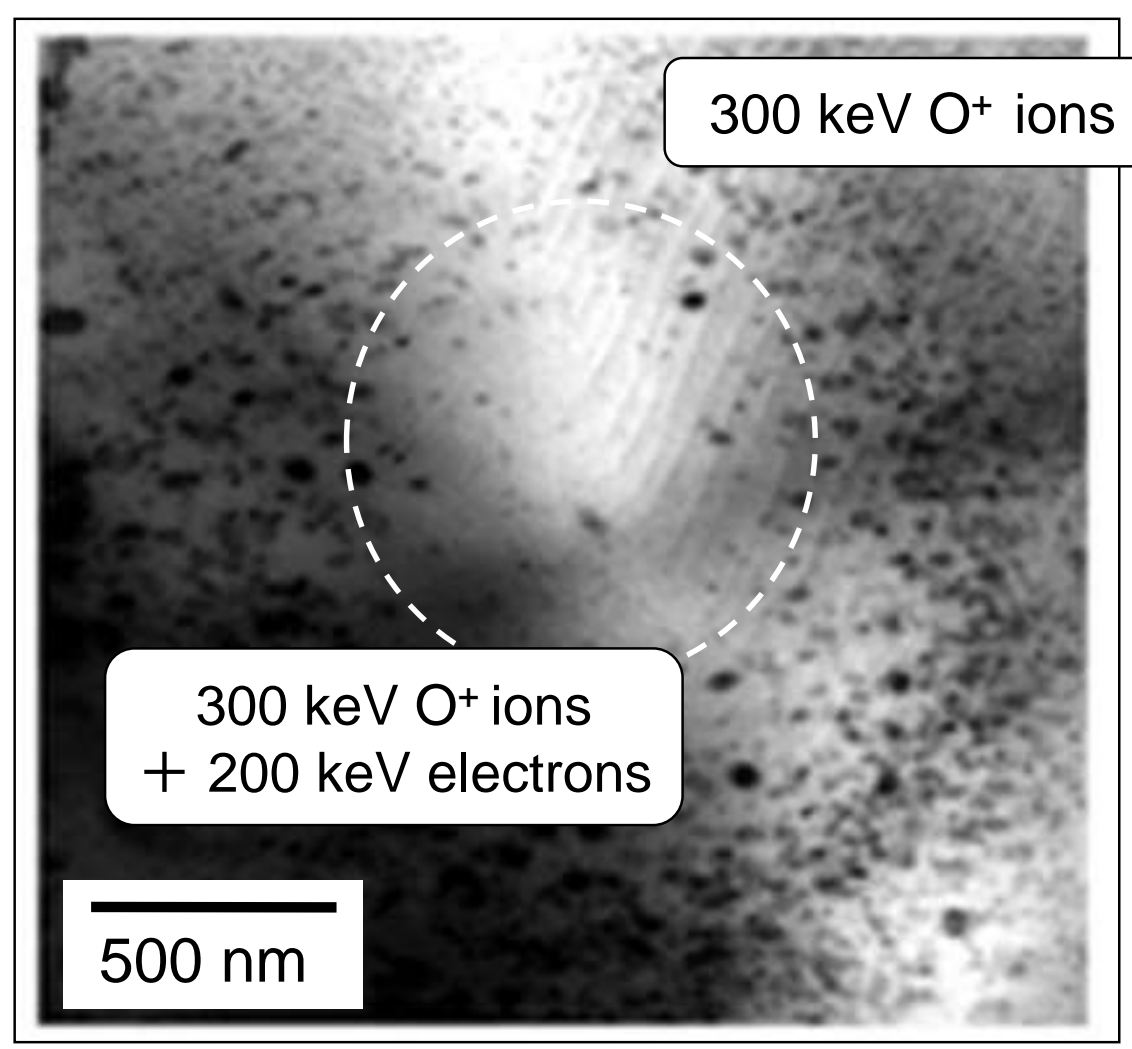

K. Yasuda, Philos Mag. 78 (1998)

$\square$ Loop formation is suppressed by electronic excitation.

․ Spinel is most sensitive to electroic exciation. 
Elimination of dislocation loops under electronic excitation $300 \mathrm{~K} \mathrm{MgAl}_{2} \mathrm{O}_{4}$ Electron flux: $7.0 \times 10^{22} \mathrm{e} / \mathrm{m}^{2} \mathrm{~s}$

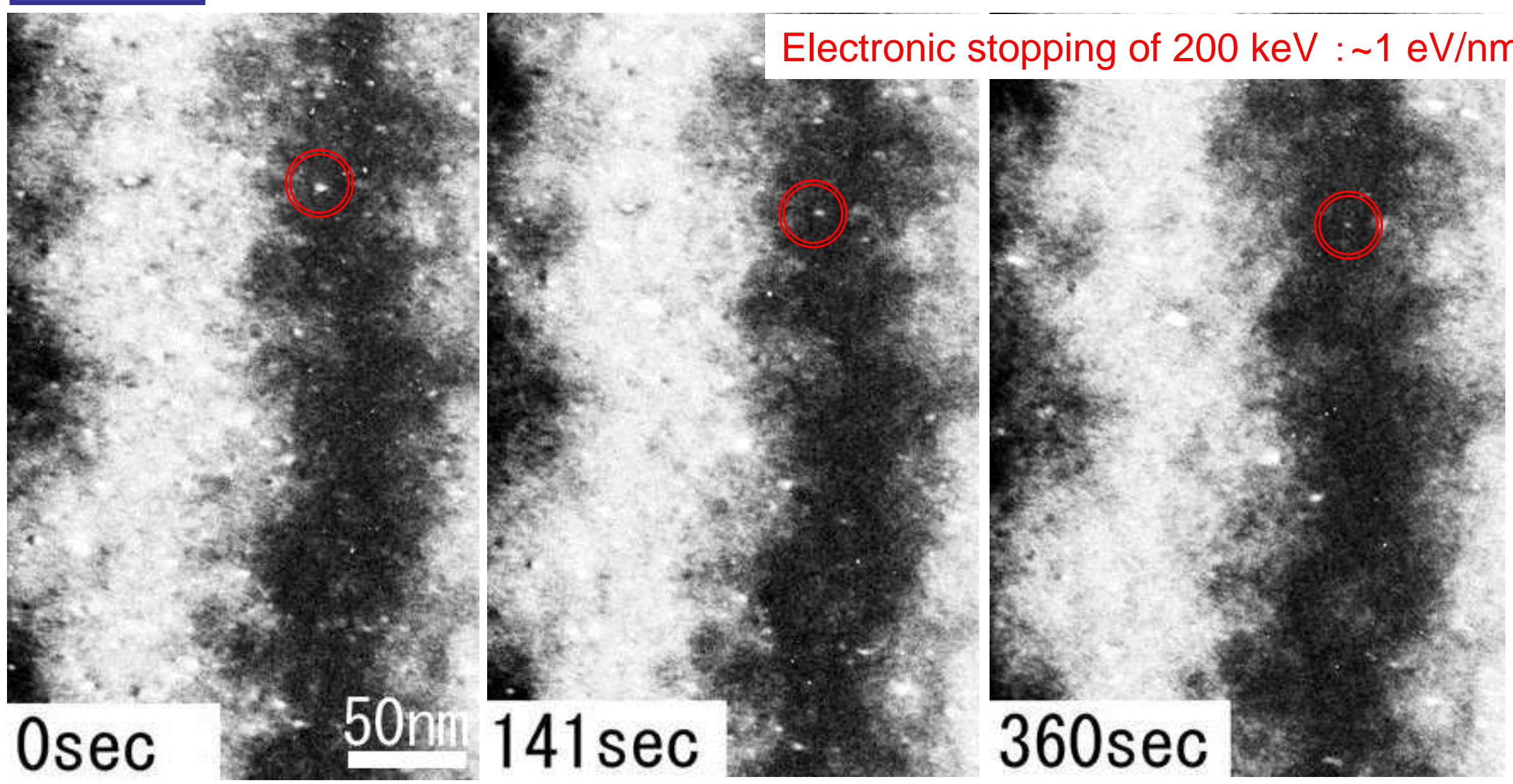

K. Yasuda NIMB 266 (2008) 2834. 
$\square$ Are

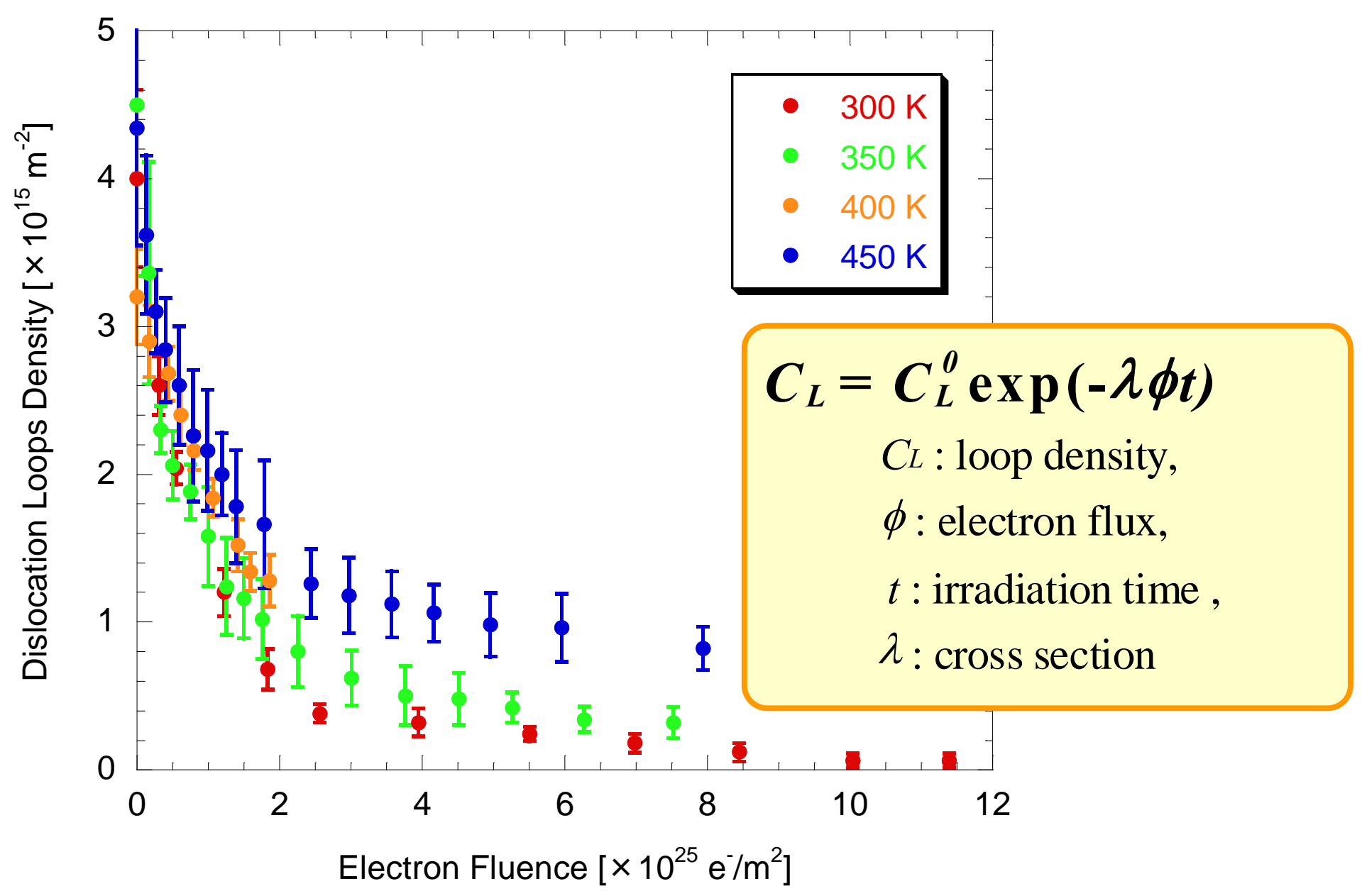

$\Rightarrow$ Evaluation of elimination cross section: $\lambda$ 


\section{$\square$ Cross section $(\lambda)$ for elimination of loops}

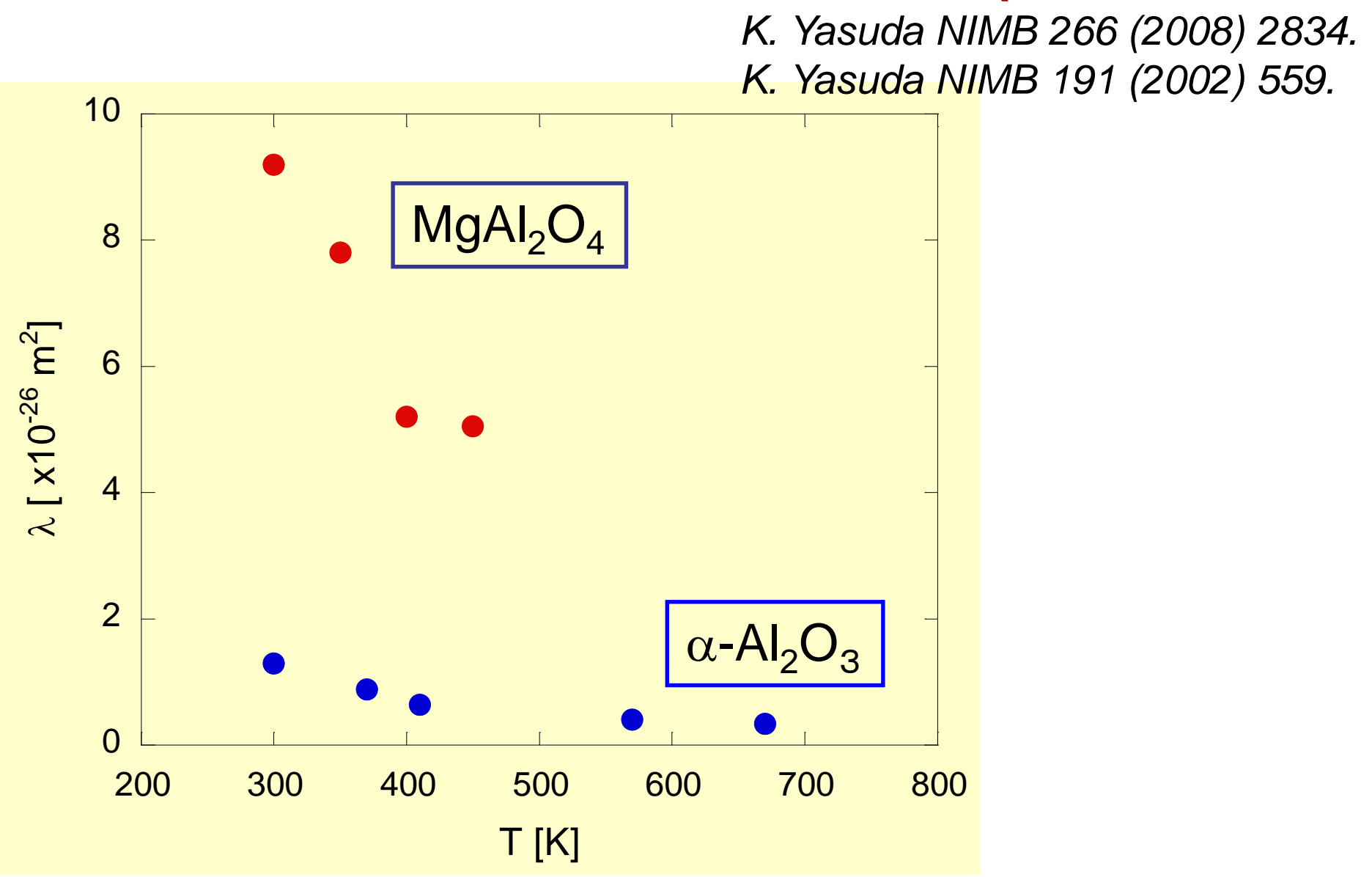

$\square$ Same temp.dependence $\Rightarrow$ same mechanism:

loops dissociate into isolated interstitials

Loops in spinel is more unstable than alumina 


\section{KYUSHU UNIVERSITY}

\section{$\square$ Size variation vs. electron fluence}
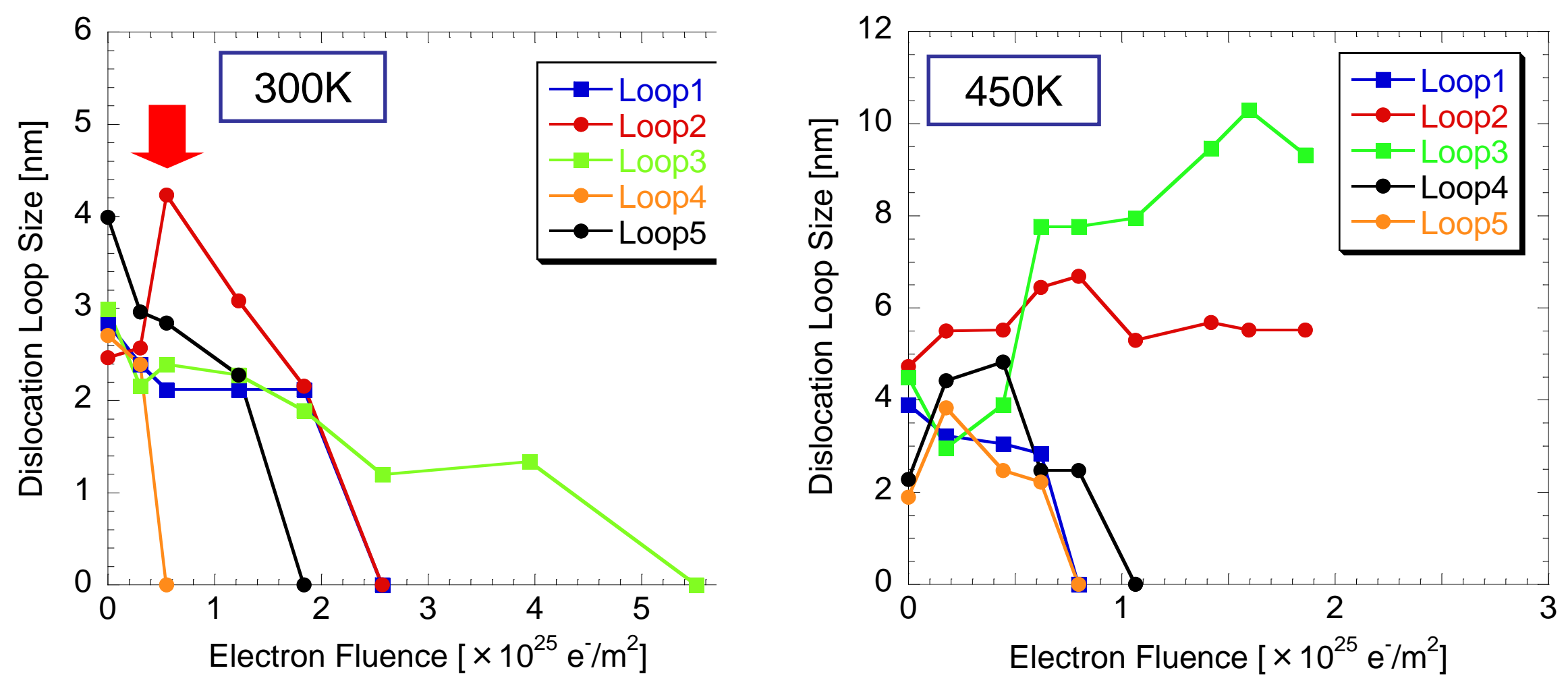


\section{$\square$ Schematic showing for the elimination of loops}

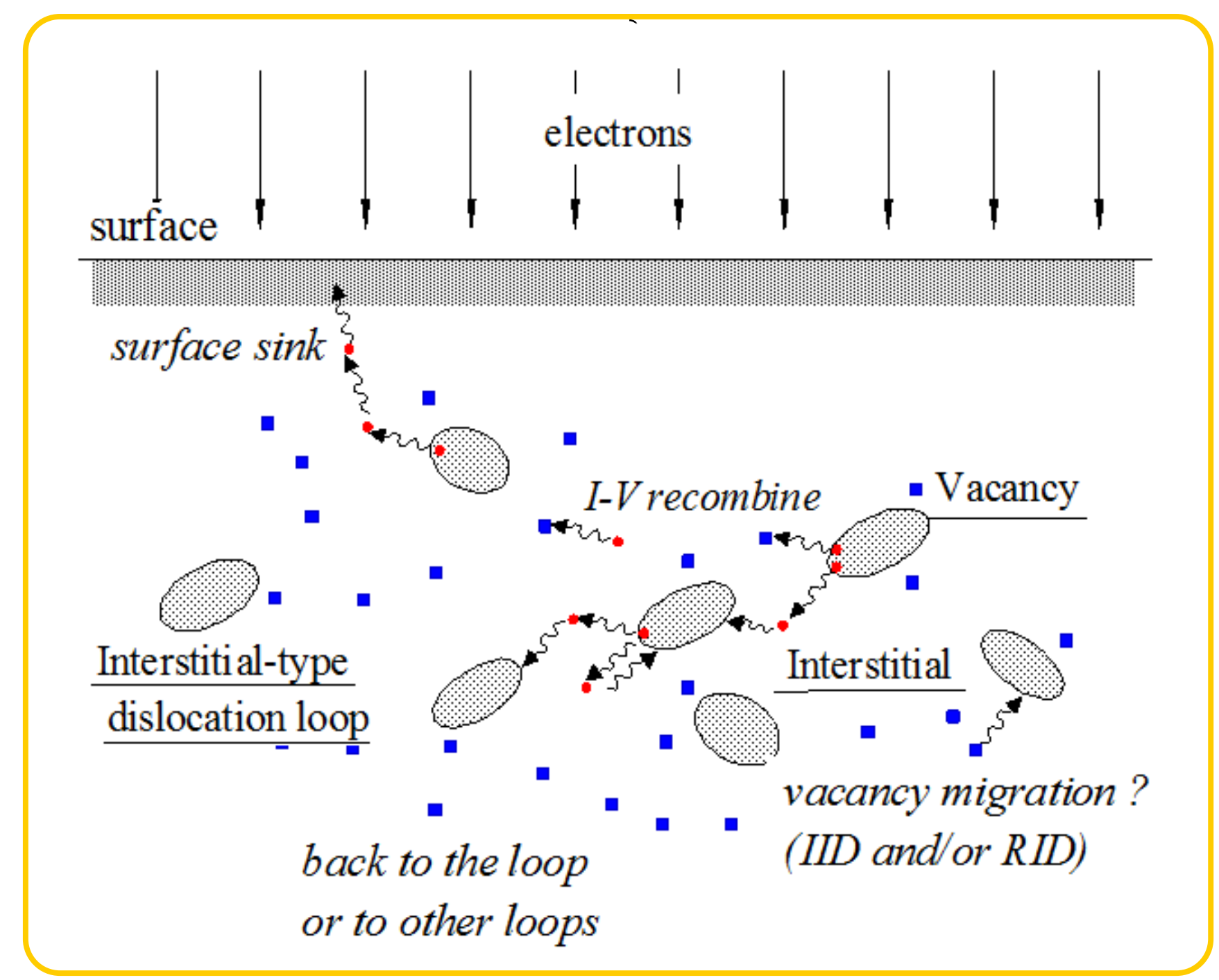




\section{Summary}

- Selective displacement damage in fluorite-type oxides:

- Charged dislocation loops with oxygen ions are formed on (111) planes

- Ion tracks in fluorite- and spinel-type oxides:

- the core region is underdensed (with vacancies).

- influence region $\sim 15 \mathrm{~nm}$ in diameter (invisible in TEM)

- interstitial generation to develop dislocation structure

- Instability of dislocation loops under electronic excitation:

- dissociate loops into isolated interstitials

- stability of loops: $\mathrm{MgAl}_{2} \mathrm{O}_{4} 4<\mathrm{Al}_{2} \mathrm{O}_{3}$ 


\section{Thank you for your attention}

